\title{
LIMIT BEHAVIOUR OF THIN INSULATING LAYERS AROUND MULTICONNECTED DOMAINS
}

\author{
Mohamed BOUTKRIDA ${ }^{\mathrm{a}}$, Nathalie GRENON ${ }^{\mathrm{b}}$, Jacqueline MOSSINO $^{\mathrm{a}}$, \\ Gonoko MOUSSA ${ }^{\mathrm{c}}$ \\ ${ }^{a}$ Ecole Normale Supérieure de Cachan, C.M.L.A. - U.M.R. 8536, 61, Avenue du Président Wilson, \\ 94235 Cachan cedex, France \\ ${ }^{\mathrm{b}}$ Faculté des Sciences, Rue Gaston Gerger, 18000 Bourges, France \\ ${ }^{\mathrm{c}}$ Ecole Normale Supérieure de Cachan and Université Henri Poincaré, Nancy I, Institut Elie Cartan, \\ BP 239, 54506 Vandoeuvre les Nancy cedex, France
}

Received 17 July 2000, revised 11 January 2001

ABSTRACT. - Let $\Omega$ be a bounded domain of $\mathbb{R}^{N}$, with boundary $\partial \Omega$. Let $\Gamma_{0}$ and $\Gamma$ be connected components of $\partial \Omega$. We assume that $\Omega$ is surrounded along $\Gamma_{0}$ and $\Gamma$ by thin insulating layers $\Sigma_{0}^{\varepsilon}$ and $\Sigma^{\varepsilon}$ of varying respective thicknesses $h_{0}^{\varepsilon}(s)$ and $h^{\varepsilon}(s), s$ being the generic point of $\Gamma_{0}$ and $\Gamma$. We denote by $\Gamma_{0}^{\varepsilon}$ and $\Gamma^{\varepsilon}$ the parts of $\partial \Sigma_{0}^{\varepsilon}$ and $\partial \Sigma^{\varepsilon}$ which do not meet $\Omega$. We consider a class of quasilinear elliptic problems with different exponents ( $p$ in $\Omega, q_{0}$ in $\Sigma_{0}^{\varepsilon}, q$ in $\Sigma^{\varepsilon}$ ) and with the following boundary conditions:

- on $\Gamma_{0}^{\varepsilon}, u^{\varepsilon}=0$,

- on $\Gamma^{\varepsilon}$, the total flux is prescribed and $u^{\varepsilon}$ is constant, but unprescribed,

- on $\Gamma_{0}$ and $\Gamma$, the natural transmission conditions.

The restricted equations in $\Sigma_{0}^{\varepsilon}$ and $\Sigma^{\varepsilon}$ have nonconstant coefficients, $\mu_{0}^{\varepsilon}$ and $\mu^{\varepsilon}$, in the form $\mu_{0}^{\varepsilon}(x)=\mu_{0}^{\varepsilon}\left(\sigma_{0}(x)\right)$ (respectively $\left.\mu^{\varepsilon}(x)=\mu^{\varepsilon}(\sigma(x))\right), \sigma_{0}$ and $\sigma$ being the respective projections on $\Gamma_{0}$ and $\Gamma$. We predict the asymptotic behaviour of this problem as $h_{0}^{\varepsilon}$ and $\mu_{0}^{\varepsilon}$ (respectively $h^{\varepsilon}$ and $\mu^{\varepsilon}$ ) tend to zero in a suitable sense, provided they are related in a convenient way.

(C) 2002 L'Association Publications de l'Institut Henri Poincaré. Published by Elsevier B.V. All rights reserved

1991 MSC: 35B40; 35J60

Keywords: Reinforcement; Boundary layer; $\Gamma$-convergence; Torsional rigidity; Quasilinear elliptic problem

RÉSUMÉ. - Soit $\Omega$ un domaine borné de $\mathbb{R}^{N}$, de frontière $\partial \Omega$. Soient $\Gamma_{0}$ et $\Gamma$ des composantes connexes de $\partial \Omega$. Nous supposons que $\Omega$ est entouré le long de $\Gamma_{0}$ et $\Gamma$ par de fines couches isolantes $\Sigma_{0}^{\varepsilon}$ et $\Sigma^{\varepsilon}$ d'épaisseurs variables respectives $h_{0}^{\varepsilon}(s)$ et $h^{\varepsilon}(s), s$ étant le point générique de $\Gamma_{0}$ ou $\Gamma$. Nous désignons par $\Gamma_{0}^{\varepsilon}$ et $\Gamma^{\varepsilon}$ les parties de $\partial \Sigma_{0}^{\varepsilon}$ et $\partial \Sigma^{\varepsilon}$ qui ne bordent pas $\Omega$. Nous considérons une classe de problèmes elliptiques quasilinéaires, avec des exposants de Sobolev différents ( $p$ dans $\Omega, q_{0}$ dans $\Sigma_{0}^{\varepsilon}, q$ dans $\Sigma^{\varepsilon}$ ) et avec les conditions au bord suivantes :

- $\operatorname{sur} \Gamma_{0}^{\varepsilon}, u^{\varepsilon}=0$, 
- $\operatorname{sur} \Gamma^{\varepsilon}$, le flux total est prescrit et $u^{\varepsilon}$ est constant, mais indéterminé,

- $\operatorname{sur} \Gamma_{0}$ et $\Gamma$, les conditions de transmission naturelles.

Les équations restreintes à $\Sigma_{0}^{\varepsilon}$ et $\Sigma^{\varepsilon}$ ont des coefficients non-constants, $\mu_{0}^{\varepsilon}$ et $\mu^{\varepsilon}$, de la forme $\mu_{0}^{\varepsilon}(x)=\mu_{0}^{\varepsilon}\left(\sigma_{0}(x)\right)\left(\right.$ resp. $\left.\mu^{\varepsilon}(x)=\mu^{\varepsilon}(\sigma(x))\right), \sigma_{0}$ et $\sigma$ étant les projections respectives sur $\Gamma_{0}$ et $\Gamma$. Nous prédisons le comportement asymptotique de ce problème, lorsque $h_{0}^{\varepsilon}$ et $\mu_{0}^{\varepsilon}$ (resp. $h^{\varepsilon}$ et $\mu^{\varepsilon}$ ) tendent vers zéro simultanément, tout en vérifiant une relation de corrélation convenable. (C) 2002 L'Association Publications de l'Institut Henri Poincaré. Published by Elsevier B.V. All rights reserved

\section{Introduction}

Let $\Omega$ be a bounded domain of $\mathbb{R}^{N}$ and let $\Gamma_{0}$ and $\Gamma$ be connected components of the boundary $\partial \Omega$ of $\Omega$. We assume that $\Omega$ is surrounded along $\Gamma_{0}$ and $\Gamma$ by thin reinforcements

$$
\begin{aligned}
& \Sigma_{0}^{\varepsilon}=\left\{s+\operatorname{tn}(s), s \in \Gamma_{0}, 0<t<h_{0}^{\varepsilon}(s)(\leqslant \varepsilon)\right\}, \\
& \Sigma^{\varepsilon}=\left\{s+\operatorname{tn}(s), s \in \Gamma, 0<t<h^{\varepsilon}(s)(\leqslant \varepsilon)\right\},
\end{aligned}
$$

where $n(s)$ denotes the outer normal to $\Omega$ at the point $s$ of $\Gamma_{0}$ or $\Gamma$. Then we denote by $\Omega^{\varepsilon}=\Omega \cup \Sigma_{0}^{\varepsilon} \cup \Sigma^{\varepsilon} \cup \Gamma_{0} \cup \Gamma$ the reinforced domain and we define $\Gamma_{0}^{\varepsilon}$ and $\Gamma^{\varepsilon}$ as the parts of $\partial \Sigma_{0}^{\varepsilon}$ and $\partial \Sigma^{\varepsilon}$ which do not meet $\Omega$. We study the limit behaviour (when $\varepsilon$ tends to zero) of some quasilinear problems with three (possibly different) exponents $p, q_{0}, q \in(1, \infty)$ of the type:

$$
\begin{cases}-\operatorname{div}\left(\left|\nabla u^{\varepsilon}\right|^{p-2} \nabla u^{\varepsilon}\right)=f^{\varepsilon} & \text { in } \Omega, \\ -\operatorname{div}\left(\mu_{0}^{\varepsilon}\left|\nabla u^{\varepsilon}\right|^{q 0}-2 \nabla u^{\varepsilon}\right)=g_{0}^{\varepsilon} & \text { in } \Sigma_{0}^{\varepsilon}, \\ -\operatorname{div}\left(\mu^{\varepsilon}\left|\nabla u^{\varepsilon}\right|^{q-2} \nabla u^{\varepsilon}\right)=g^{\varepsilon} & \text { in } \Sigma^{\varepsilon}, \\ u^{\varepsilon}=0 & \text { on } \Gamma_{0}^{\varepsilon}=\left(\partial \Sigma_{0}^{\varepsilon}\right) \backslash \Gamma_{0}, \\ u^{\varepsilon} \text { is constant (undetermined) } & \text { on } \Gamma^{\varepsilon}=\left(\partial \Sigma^{\varepsilon}\right) \backslash \Gamma, \\ \int_{\Gamma^{\varepsilon}} \mu^{\varepsilon}\left|\nabla u^{\varepsilon}\right|^{q-2} \frac{\partial u^{\varepsilon}}{\partial n} \mathrm{~d} s=I^{\varepsilon} & \text { (given), } \\ \frac{\partial u^{\varepsilon}}{\partial n}=0 & \text { on } \partial \Omega \backslash\left(\Gamma_{0}^{\varepsilon} \cup \Gamma^{\varepsilon}\right), \\ + \text { transmission conditions } & \text { on } \Gamma_{0} \text { and } \Gamma,\end{cases}
$$

where we write "transmission conditions" for:

$$
\begin{gathered}
u_{\mid \Omega}^{\varepsilon}= \begin{cases}u_{\mid \Sigma_{0}^{\varepsilon}}^{\varepsilon} & \text { on } \Gamma_{0}, \\
u_{\mid \Sigma^{\varepsilon}}^{\varepsilon} & \text { on } \Gamma,\end{cases} \\
\left|\nabla u_{|\Omega|}^{\varepsilon}\right|^{p-2} \frac{\partial\left(u_{\mid \Omega}^{\varepsilon}\right)}{\partial n}= \begin{cases}\mu^{\varepsilon}\left|\nabla u_{\mid \Sigma_{0}^{\varepsilon}}^{\varepsilon}\right|^{q 0-2} \frac{\partial\left(u_{\mid \Sigma_{0}^{\varepsilon}}^{\varepsilon}\right)}{\partial n} & \text { on } \Gamma_{0}, \\
\mu^{\varepsilon}\left|\nabla u_{\mid \Sigma^{\varepsilon}}^{\varepsilon}\right|^{q-2} \frac{\partial\left(u_{\mid \Sigma^{\varepsilon}}^{\varepsilon}\right)}{\partial n} & \text { on } \Gamma,\end{cases}
\end{gathered}
$$


and where $n$ denotes the outer normal to $\partial \Omega$ or $\partial \Omega^{\varepsilon}$. In an electric (or heat propagation) setting, if $\Omega^{\varepsilon}$ is doubly connected, with $\Gamma^{\varepsilon}$ as inner part of the boundary $\partial \Omega^{\varepsilon}$ and $\Gamma_{0}^{\varepsilon}$ as outer part, the boundary constraints mean that the potential (or temperature) has a given value on $\Gamma_{0}^{\varepsilon}$ and that $\Gamma^{\varepsilon}$ surrounds a perfect conductor, in which consequently the potential (or temperature) is constant, but unprescribed. The total flux, i.e. $I^{\varepsilon}$, is then given in terms of the integral of the source term over the perfectly conducting region. The assumption that $\mu_{0}^{\varepsilon}$ and $\mu^{\varepsilon}$ are small means that $\Sigma_{0}^{\varepsilon}$ and $\Sigma^{\varepsilon}$ modelize (unperfect) insulating layers. The torsional rigidity problem for a cable of multiconnected cross section also is of the same form.

To be more explicit, consider $\mathcal{D}^{\varepsilon}$ such that $\partial \mathcal{D}^{\varepsilon}=\Gamma_{0}^{\varepsilon}$ and $\mathcal{C}^{\varepsilon}$ such that $\partial \mathcal{C}^{\varepsilon}=\Gamma^{\varepsilon}$ $\left(\mathcal{D}^{\varepsilon}=\Omega^{\varepsilon} \cup \overline{\mathcal{C}^{\varepsilon}}\right)$; the model problem consists in minimizing the energy

$$
\mathcal{E}^{\varepsilon}(v)=\int_{\mathcal{D}^{\varepsilon}} c^{\varepsilon}|\nabla v|^{2} \mathrm{~d} x-\int_{\mathcal{D}^{\varepsilon}} f^{\varepsilon} v \mathrm{~d} x,
$$

over the subset of functions $v$ in $H_{0}^{1}\left(\mathcal{D}^{\varepsilon}\right)$ such that $\nabla v=0$ in $\mathcal{C}^{\varepsilon}$, with a conductivity coefficient $c^{\varepsilon}$ having value 1 in $\Omega, \mu^{\varepsilon}$ in $\Sigma^{\varepsilon}, \mu_{0}^{\varepsilon}$ in $\Sigma_{0}^{\varepsilon}$. This problem is equivalent to minimizing

$$
\mathcal{E}^{\varepsilon}(v)=\int_{\Omega^{\varepsilon}} c^{\varepsilon}|\nabla v|^{2} \mathrm{~d} x-\int_{\Omega^{\varepsilon}} f^{\varepsilon} v \mathrm{~d} x-v_{\mid \Gamma^{\varepsilon}} \int_{\mathcal{C}^{\varepsilon}} f^{\varepsilon} \mathrm{d} x,
$$

over the subset of functions $v$ in $H^{1}\left(\Omega^{\varepsilon}\right)$ such that $v=0$ on $\Gamma_{0}^{\varepsilon}$ and $v$ is constant (but unprescribed) on $\Gamma^{\varepsilon}$. Now the Euler equation of the above minimization problem is

$$
\begin{cases}-\Delta u^{\varepsilon}=f^{\varepsilon} & \text { in } \Omega, \\ -\operatorname{div}\left(\mu_{0}^{\varepsilon} \nabla u^{\varepsilon}\right)=f^{\varepsilon} & \text { in } \Sigma_{0}^{\varepsilon}, \\ -\operatorname{div}\left(\mu^{\varepsilon} \nabla u^{\varepsilon}\right)=f^{\varepsilon} & \text { in } \Sigma^{\varepsilon}, \\ u^{\varepsilon}=0 & \text { on } \Gamma_{0}^{\varepsilon}, \\ u^{\varepsilon} \text { is constant (undetermined) } & \text { on } \Gamma^{\varepsilon}, \\ \int_{\Gamma^{\varepsilon}} \mu^{\varepsilon} \frac{\partial u^{\varepsilon}}{\partial n} \mathrm{~d} s=\int_{\mathcal{C}^{\varepsilon}} f^{\varepsilon} \mathrm{d} x, & \\ u_{\mid \Omega}^{\varepsilon}=u_{\mid \Sigma_{0}^{\varepsilon}}^{\varepsilon} \text { and } \quad \frac{\partial u_{\mid \Omega}^{\varepsilon}}{\partial n}=\mu_{0}^{\varepsilon} \frac{\partial\left(u_{\mid \Sigma^{\varepsilon}}^{\varepsilon}\right)}{\partial n} & \text { on } \Gamma_{0}, \\ u_{\mid \Omega}^{\varepsilon}=u_{\mid \Sigma^{\varepsilon}}^{\varepsilon} \text { and } \quad \frac{\partial u_{\mid \Omega}^{\varepsilon}}{\partial n}=\mu^{\varepsilon} \frac{\partial\left(u_{\mid \Sigma^{\varepsilon}}^{\varepsilon}\right)}{\partial n} & \text { on } \Gamma,\end{cases}
$$

which is the model equation for $(\mathcal{E})$.

Similar problems (not involving $\Sigma^{\varepsilon}$ ) were considered by Boutkrida, Mossino and Moussa in [4] and [5]. Previous works on reinforcement problems were done by Sanchez-Palencia [13,14], Acerbi and Buttazzo [1], Brezis, Caffarelli and Friedman [6], Buttazzo and Kohn [7] and by Buttazzo, Dal Maso and Mosco [8]. To our knowledge, the condition on the given flux and the undetermined constant boundary value appeared only very recently in this context of boundary layers [11].

As in [4] and [5] the shape of the reinforcement may depend on $\varepsilon$, that is we consider "general" functions $h_{0}^{\varepsilon}$ and $h^{\varepsilon}$, and the insulating (or reinforcing) material may be 
inhomogeneous along $\Gamma_{0}$ and $\Gamma$, but $\mu_{0}^{\varepsilon}$ and $\mu^{\varepsilon}$ are constant along each normal to $\Gamma_{0}$ and $\Gamma$ : in other words $\mu_{0}^{\varepsilon}(x)=\mu_{0}^{\varepsilon}\left(\sigma_{0}(x)\right)$ (respectively $\mu^{\varepsilon}(x)=\mu^{\varepsilon}(\sigma(x))$ ), where $\sigma_{0}(x)$ (respectively $\sigma(x)$ ) denotes the projection of $x \in \Sigma_{0}^{\varepsilon}$ (respectively $\Sigma^{\varepsilon}$ ) on $\Gamma_{0}$ (respectively $\Gamma$ ). We define $a_{0}^{\varepsilon}: \Gamma_{0} \rightarrow \mathbb{R}$ (respectively $a^{\varepsilon}: \Gamma \rightarrow \mathbb{R}$ ) by $a_{0}^{\varepsilon}=\mu_{0}^{\varepsilon}\left(h_{0}^{\varepsilon}\right)^{1-q_{0}}$ (respectively $a^{\varepsilon}=\mu^{\varepsilon}\left(h^{\varepsilon}\right)^{1-q}$ ). We assume essentially that $a_{0}^{\varepsilon}$ are positive functions in $L^{\infty}\left(\Gamma_{0}\right)$ (and the same for $a^{\varepsilon} \in L^{\infty}(\Gamma)$ ), with uniformly bounded inverses, and that $\Gamma_{0}$ (respectively $\Gamma$ ) is divided into two parts:

- $\underline{\Gamma}_{0}\left(\right.$ respectively $\underline{\Gamma}$ ) such that either $\underline{\Gamma}_{0}$ (respectively $\underline{\Gamma}$ ) is empty or $\frac{1}{a_{0}^{\varepsilon}}$ tends to zero in $L^{q_{0}^{\prime}-1}\left(\underline{\Gamma}_{0}\right)$ (respectively $\frac{1}{a^{\varepsilon}} \rightarrow 0$ in $\left.L^{q^{\prime}-1}(\underline{\Gamma})\right), q_{0}^{\prime}$ and $q^{\prime}$ denoting the conjugates of $q_{0}$ and $q$ (e.g. $\frac{1}{q}+\frac{1}{q^{\prime}}=1$ ),

- $\bar{\Gamma}_{0}$ (respectively $\bar{\Gamma}$ ) such that either $\bar{\Gamma}_{0}$ (respectively $\bar{\Gamma}$ ) is empty or $a_{0}^{\varepsilon}$ tends to $a_{0}$ in weak $\star-L^{\infty}\left(\bar{\Gamma}_{0}\right)$ (respectively $a^{\varepsilon} \rightarrow a$ in weak $\star-L^{\infty}(\bar{\Gamma})$ ) and such that $h_{0}^{\varepsilon}$ (respectively $h^{\varepsilon}$ ) does not oscillate too much on $\bar{\Gamma}_{0}$ (respectively $\bar{\Gamma}$ ).

We prove that the limit problem has the form

$$
\begin{cases}-\operatorname{div}\left(|\nabla u|^{p-2} \nabla u\right)=f & \text { in } \Omega, \\ u=0 & \text { on } \underline{\Gamma}_{0}, \\ |\nabla u|^{p-2} \frac{\partial u}{\partial n}+a_{0}|u|^{q_{0}-2} u=0 & \text { on } \bar{\Gamma}_{0}, \\ u=k \text { (undetermined constant) } & \text { on } \underline{\Gamma}, \\ |\nabla u|^{p-2} \frac{\partial u}{\partial n}+a|u-k|^{q-2}(u-k)=0 & \text { on } \bar{\Gamma}, \\ \int_{\Gamma}|\nabla u|^{p-2} \frac{\partial u}{\partial n} \mathrm{~d} s=I, & \\ \frac{\partial u}{\partial n}=0 & \text { on } \partial \Omega \backslash\left(\Gamma_{0} \cup \Gamma\right),\end{cases}
$$

where $f$ and $I$ are respective limits of $f^{\varepsilon}$ and $I^{\varepsilon}$. As Acerbi and Buttazzo did in [1], we use the $\Gamma$-convergence theory introduced by De Giorgi [10] (see also Attouch [2] and Dal Maso [9]) and we actually are able to predict the explicit limit of more general minimization problems than those associated with $(1.1),(1.2)$ and $(\mathcal{E})$ :

- One can introduce additional constraints on $u_{\mid \Omega}^{\varepsilon}$;

- The reinforcements (1.1) and (1.2) can be generalized to

$$
\begin{gathered}
\Sigma_{0}^{\varepsilon}=\left\{s+t n_{H_{0}}(s), s \in \Gamma_{0}, 0<t<h_{0}^{\varepsilon}(s)(\leqslant \varepsilon)\right\}, \\
\Sigma^{\varepsilon}=\left\{s+t n_{H}(s), s \in \Gamma, 0<t<h^{\varepsilon}(s)(\leqslant \varepsilon)\right\},
\end{gathered}
$$

where $n_{H_{0}}(s)$ (respectively $n_{H}(s)$ ) is supported by the line of points having $H_{0}$ (respectively $H$ )-projection $s$ on $\Gamma_{0}$ (respectively $\Gamma$ ) and where $H_{0}$ and $H$ are general norms $\left(n_{H}=n\right.$, the unit normal vector, if $H$ is the euclidian norm);

- In the energy functional, the term

$$
\frac{1}{p} \int_{\Omega}|\nabla v|^{p} \mathrm{~d} x+\frac{1}{q_{0}} \int_{\Sigma_{0}^{\varepsilon}} \mu_{0}^{\varepsilon} \circ \sigma_{0}|\nabla v|^{q_{0}} \mathrm{~d} x+\frac{1}{q} \int_{\Sigma^{\varepsilon}} \mu^{\varepsilon} \circ \sigma|\nabla v|^{q} \mathrm{~d} x
$$


can be generalized to the anisotropic one

$$
F\left(v_{\mid \Omega}\right)+\int_{\Sigma_{0}^{\varepsilon}} \frac{a_{0}^{\varepsilon} \circ \sigma_{0}}{h_{0}^{\varepsilon} \circ \sigma_{0}} G_{0}\left(h_{0}^{\varepsilon} \circ \sigma_{0} H_{0}^{o}(\nabla v)\right) \mathrm{d} x+\int_{\Sigma^{\varepsilon}} \frac{a^{\varepsilon} \circ \sigma}{h^{\varepsilon} \circ \sigma} G\left(h^{\varepsilon} \circ \sigma H^{o}(\nabla v)\right) \mathrm{d} x,
$$

where (e.g.) $\left(\mu^{\varepsilon} \circ \sigma\right)(x)=\mu^{\varepsilon}(\sigma(x)), \sigma(x)$ (respectively $\left.\sigma_{0}(x)\right)$ is the projection of $x$ for the $H$-norm (respectively $H_{0}$-norm) and where (e.g.) $H^{o}$ denotes the dual norm of $H$, defined as

$$
H^{o}\left(\xi^{o}\right)=\sup _{\xi \neq 0} \frac{\xi^{o} \cdot \xi}{H(\xi)} .
$$

We give the precise assumptions on $F, G, G_{0}, H, H_{0}$ in the following.

We would like to emphasize the connection between the geometry of the reinforcements $\Sigma^{\varepsilon}, \Sigma_{0}^{\varepsilon}$ (defined by $H$ and $H_{0}$ ) and the energy functional, whose integrands on $\Sigma^{\varepsilon}$ and $\Sigma_{0}^{\varepsilon}$ are defined in terms of the dual norms $H^{o}$ and $H_{0}^{o}$.

Finally, let us mention that the result can be easily generalized to reinforcements along a finite number of components of $\partial \Omega$.

\section{Statement of the problem and of the result}

Let $H: \mathbb{R}^{N} \rightarrow \mathbb{R}^{+}$be a norm. In particular

$$
\begin{gathered}
\forall t \in \mathbb{R}, \forall \xi \in \mathbb{R}^{N}, \quad H(t \xi)=|t| H(\xi), \\
\exists \delta_{1}, \delta_{2}>0, \forall \xi \in \mathbb{R}^{N}, \quad \delta_{1}|\xi| \leqslant H(\xi) \leqslant \delta_{2}|\xi|, \\
\forall \xi_{1}, \xi_{2} \in \mathbb{R}^{N}, \quad H\left(\xi_{1}+\xi_{2}\right) \leqslant H\left(\xi_{1}\right)+H\left(\xi_{2}\right)
\end{gathered}
$$

and from (2.1), (2.3), $H$ is convex. The dual function of $H$, defined as

$$
H^{o}\left(\xi^{o}\right)=\sup \left\{\frac{\xi^{o} . \xi}{H(\xi)}, \xi \in \mathbb{R}^{N}, \xi \neq 0\right\}=\sup \left\{\xi^{o} . \xi, \xi \in \mathbb{R}^{N}, 0<H(\xi) \leqslant 1\right\}
$$

is also a norm, with

$$
\forall \xi^{o} \in \mathbb{R}^{N}, \quad \frac{1}{\delta_{2}}\left|\xi^{o}\right| \leqslant H^{o}\left(\xi^{o}\right) \leqslant \frac{1}{\delta_{1}}\left|\xi^{o}\right| ;
$$

$H$ and $H^{o}$ are dual to each other and satisfy

$$
\forall \xi \in \mathbb{R}^{N}, \forall \xi^{o} \in \mathbb{R}^{N}, \quad\left|\xi^{o} . \xi\right| \leqslant H(\xi) H^{o}\left(\xi^{o}\right) ;
$$

In this paper we consider two such norms $H$ and $H_{0}$; we assume that they are differentiable at any point but zero and that $H^{o}$ and $H_{0}^{o}$ are strictly convex.

Now consider a bounded regular domain $\Omega$ in $\mathbb{R}^{N}$ and let $\Gamma$ and $\Gamma_{0}$ be connected components of $\partial \Omega$. We introduce the distances (related to $H$ and $H_{0}$ ) from $x$ to $\Gamma$ and $\Gamma_{0}$, defined by

$$
t(x)=\min _{s \in \Gamma} H(x-s), \quad t_{0}(x)=\min _{s \in \Gamma_{0}} H_{0}(x-s) .
$$


Let us remark that the above minima are achieved. Moreover if, for example, $\sigma$ minimizes $H(x-s)$ for $s \in \Gamma$ and if $y$ is on the segment $[\sigma, x]$, then $\sigma$ also minimizes $H(y-s)$ for $s \in \Gamma$. (Actually assume $\sigma^{\prime}$ is on $\Gamma$ with $H\left(y-\sigma^{\prime}\right)<H(y-\sigma)$. Then $H\left(x-\sigma^{\prime}\right) \leqslant H(x-y)+H\left(y-\sigma^{\prime}\right)<H(x-y)+H(y-\sigma)=H(x-\sigma)$ and we get a contradiction.) It follows that the set of points having $H$-projection $\sigma$ on $\Gamma$ is a line emanating from $\sigma$.

Let $\Omega^{\prime} \supset \Omega$ be the domain having boundary $\left(\partial \Omega \backslash\left(\Gamma \cup \Gamma_{0}\right)\right) \cup \Gamma^{\prime} \cup \Gamma_{0}^{\prime}$, with $\Gamma^{\prime}$ and $\Gamma_{0}^{\prime}$ at given small distance $t^{\prime}$ (relative to $H$ and $H_{0}$ respectively) from $\Gamma$ and $\Gamma_{0}$. We have $\Omega^{\prime} \backslash \bar{\Omega}=\Sigma^{\prime} \cup \Sigma_{0}^{\prime}$ and we assume that $\Sigma^{\prime}$ and $\Sigma_{0}^{\prime}$ are $\mathcal{C}^{1}$-diffeomorphic to $\Gamma \times\left(0, t^{\prime}\right)$ and $\Gamma_{0} \times\left(0, t^{\prime}\right)$ by the mappings $\mathcal{D}$ and $\mathcal{D}_{0}$ :

$$
\mathcal{D}: x \in \Sigma^{\prime} \rightarrow(\sigma(x), t(x)) \in \Gamma \times\left(0, t^{\prime}\right),
$$

with $t(x)=\min _{s \in \Gamma} H(x-s)$ as above and $\sigma(x)=\arg \min _{s \in \Gamma} H(x-s)$. $\left(\mathcal{D}_{0}\right.$ is defined similarly). We notice that (e.g.) $\mathcal{D}^{-1}(\sigma, t)=\sigma+t n_{H}(\sigma)$ with $H\left(n_{H}(\sigma)\right)=1$ (since $\left.t=H\left(t n_{H}(\sigma)\right)\right)$.

Let $\varepsilon<t^{\prime}$ be a small parameter (hereafter $\varepsilon$ will describe a sequence of positive numbers tending to zero) and let $h^{\varepsilon}: \Gamma \rightarrow \mathbb{R}^{+} \backslash\{0\}$ be a positive $\mathcal{C}^{1}$-function such that

$$
\forall \sigma \in \Gamma, \quad h^{\varepsilon}(\sigma) \leqslant \varepsilon ;
$$

$h^{\varepsilon}$ defines the reinforcement $\Sigma^{\varepsilon}$ of $\Omega$ along $\Gamma$ :

$$
\Sigma^{\varepsilon}=\left\{x \in \Sigma^{\prime}, 0<t(x)<h^{\varepsilon}(\sigma(x))\right\}=\left\{\sigma+t n_{H}(\sigma), \sigma \in \Gamma, 0<t<h^{\varepsilon}(\sigma)\right\} .
$$

We set

$$
\Gamma^{\varepsilon}=\left\{\sigma+h^{\varepsilon}(\sigma) n_{H}(\sigma), \sigma \in \Gamma\right\} .
$$

We consider also a similar function $h_{0}^{\varepsilon}$ defined on $\Gamma_{0}$ and we associate with it the reinforcement $\Sigma_{0}^{\varepsilon}$ (of $\Omega$ along $\Gamma_{0}$ ) and the part $\Gamma_{0}^{\varepsilon}$ of its boundary which does not meet $\Omega$. We denote by $\Omega^{\varepsilon}=\Omega \cup \Sigma^{\varepsilon} \cup \Sigma_{0}^{\varepsilon} \cup \Gamma \cup \Gamma_{0}$ the reinforced domain. Note that $\Omega \subset \Omega^{\varepsilon} \subset \Omega^{\prime}=\Omega \cup \Sigma^{\prime} \cup \Sigma_{0}^{\prime} \cup \Gamma \cup \Gamma_{0}$.

With the above geometrical data and given $p, q, q_{0}$ in $(1, \infty)$, we consider the functional space

$$
\begin{aligned}
V^{\varepsilon}=\left\{v: \Omega^{\varepsilon} \rightarrow \mathbb{R}, v_{\mid \Omega} \in W^{1, p}(\Omega), v_{\mid \Sigma^{\varepsilon}} \in W^{1, q}\left(\Sigma^{\varepsilon}\right), v_{\mid \Sigma_{0}^{\varepsilon}} \in W^{1, q_{0}}\left(\Sigma_{0}^{\varepsilon}\right),\right. \\
\\
\left.v_{\mid \Omega}=v_{\mid \Sigma^{\varepsilon}} \text { on } \Gamma, v_{\mid \Omega}=v_{\mid \Sigma_{0}^{\varepsilon}} \text { on } \Gamma_{0}, v_{\mid \Gamma^{\varepsilon}}=\text { (undetermined) constant, } v_{\mid \Gamma_{0}^{\varepsilon}}=0\right\} .
\end{aligned}
$$

We are given data $I^{\varepsilon}, f^{\varepsilon}, g^{\varepsilon}, g_{0}^{\varepsilon}, F, K, G, G_{0}, a^{\varepsilon}, a_{0}^{\varepsilon}$ such that

- $I^{\varepsilon} \in \mathbb{R}, f^{\varepsilon} \in L^{p^{\prime}}(\Omega), g^{\varepsilon} \in L^{q^{\prime}}\left(\Sigma^{\varepsilon}\right), g_{0}^{\varepsilon} \in L^{q_{0}^{\prime}}\left(\Sigma_{0}^{\varepsilon}\right)$, with $p^{\prime}, q^{\prime}$ and $q_{0}^{\prime}$ the conjugates of $p, q$ and $q_{0}$,

- $F: W^{1, p}(\Omega) \rightarrow \mathbb{R}$ is a continuous strictly convex functional such that

$$
\exists \lambda>0, \exists \lambda^{\prime}>0, \forall v \in W^{1, p}(\Omega), \quad F(v) \geqslant \lambda\|\nabla v\|_{L^{p}(\Omega)^{N}}^{p}-\lambda^{\prime}\|v\|_{W^{1, p}(\Omega)},
$$

- $K$ is a nonempty closed convex subset of $W^{1, p}(\Omega)$, corresponding to conditions that concern neither $\Gamma$ nor $\Gamma_{0}$, 
- $G$ and $G_{0}: \mathbb{R}^{+} \rightarrow \mathbb{R}^{+}$are increasing, strictly convex functions and

$$
\exists \mu_{1}, \mu_{2}>0, \forall \eta \in \mathbb{R}^{+}, \quad \mu_{1} \eta^{q} \leqslant G(\eta) \leqslant \mu_{2} \eta^{q}
$$

(respectively $\mu_{1} \eta^{q_{0}} \leqslant G_{0}(\eta) \leqslant \mu_{2} \eta^{q_{0}}$ ),

- $a^{\varepsilon} \in L^{\infty}(\Gamma), a^{\varepsilon}>0$ a.e. and $\frac{1}{a^{\varepsilon}} \in L^{\infty}(\Gamma), a_{0}^{\varepsilon} \in L^{\infty}\left(\Gamma_{0}\right), a_{0}^{\varepsilon}>0$ a.e. and $\frac{1}{a_{0}^{\varepsilon}} \in$ $L^{\infty}\left(\Gamma_{0}\right)$.

Now we are able to define $J^{\varepsilon}: V^{\varepsilon} \rightarrow \mathbb{R}$ by

$$
\begin{aligned}
J^{\varepsilon}(v)= & F\left(v_{\mid \Omega}\right)+\int_{\Sigma^{\varepsilon}} \frac{a^{\varepsilon} \circ \sigma}{h^{\varepsilon} \circ \sigma} G\left(h^{\varepsilon} \circ \sigma H^{o}(\nabla v)\right) \mathrm{d} x+\int_{\Sigma_{0}^{\varepsilon}} \frac{a_{0}^{\varepsilon} \circ \sigma_{0}}{h_{0}^{\varepsilon} \circ \sigma_{0}} G_{0}\left(h_{0}^{\varepsilon} \circ \sigma_{0} H_{0}^{o}(\nabla v)\right) \mathrm{d} x \\
& -\int_{\Omega} f^{\varepsilon} v \mathrm{~d} x-\int_{\Sigma^{\varepsilon}} g^{\varepsilon} v \mathrm{~d} x-\int_{\Sigma_{0}^{\varepsilon}} g_{0}^{\varepsilon} v \mathrm{~d} x-I^{\varepsilon} v_{\mid \Gamma^{\varepsilon}},
\end{aligned}
$$

where (e.g.) $\left(a^{\varepsilon} \circ \sigma\right)(x)=a^{\varepsilon}(\sigma(x))$ and the first integral over $\Sigma^{\varepsilon}$ is meaningful since by (2.5), (2.6), (2.8) the nonnegative integrand is bounded by $\mu_{2} \varepsilon^{q-1} \delta_{1}^{-q}|| a^{\varepsilon} \|_{L^{\infty}(\Gamma)}|\nabla v|^{q}$.

Our aim is to study the limit, as $\varepsilon$ tends to zero, of the sequence of minimization problems

$$
\operatorname{Inf}\left\{J^{\varepsilon}(v), v \in V^{\varepsilon}, v_{\mid \Omega} \in K\right\} .
$$

Proposition 1. $-\left(\mathcal{P}^{\varepsilon}\right)$ has a unique solution $u^{\varepsilon}$.

Proof. - In this proof, as well as in the whole paper, $C$ denotes various constants and we write $C^{\varepsilon}$ for constants depending on $\varepsilon$. It follows from (2.5), (2.8) and the Lebesgue dominated convergence theorem that $v \rightarrow G\left(h^{\varepsilon} \circ \sigma H^{o}(\nabla v)\right)$ is continuous from $W^{1, q}\left(\Sigma^{\varepsilon}\right)$ to $L^{1}\left(\Sigma^{\varepsilon}\right)$, so that the first integral over $\Sigma^{\varepsilon}$ is a continuous function on $W^{1, q}\left(\Sigma^{\varepsilon}\right)$. The same is true for $\Sigma_{0}^{\varepsilon}$.

Let us prove that

$$
\|v\|_{V^{\varepsilon}}=\|\nabla v\|_{L^{p}(\Omega)^{N}}+\|\nabla v\|_{L^{q}\left(\Sigma^{\varepsilon}\right)^{N}}+\|\nabla v\|_{L^{q_{0}}\left(\Sigma_{0}^{\varepsilon}\right)^{N}}
$$

is a norm on $V^{\varepsilon}$ which is equivalent to the usual one induced by $W^{1, p}(\Omega) \times W^{1, q}\left(\Sigma^{\varepsilon}\right) \times$ $W^{1, q_{0}}\left(\Sigma_{0}^{\varepsilon}\right)$. Actually by Poincaré inequality

$$
\|v\|_{W^{1, q_{0}}\left(\Sigma_{0}^{\varepsilon}\right)} \leqslant C^{\varepsilon}\|\nabla v\|_{L^{q_{0}\left(\Sigma_{0}^{\varepsilon}\right)^{N}}}
$$

and

$$
\begin{gathered}
\|v\|_{L^{p}(\Omega)} \leqslant C\left[\|\nabla v\|_{L^{p}(\Omega)^{N}}+\left\|v_{\mid \Gamma_{0}}\right\|_{L^{1}\left(\Gamma_{0}\right)}\right] \\
\|v\|_{L^{q}\left(\Sigma^{\varepsilon}\right)} \leqslant C^{\varepsilon}\left[\|\nabla v\|_{L^{q}\left(\Sigma^{\varepsilon}\right)^{N}}+\left\|v_{\mid \Gamma}\right\|_{\left.L^{1}(\Gamma)\right)}\right] .
\end{gathered}
$$

Moreover

$$
\begin{gathered}
\left\|v_{\mid \Gamma_{0}}\right\|_{L^{1}\left(\Gamma_{0}\right)} \leqslant C\left\|v_{\mid \Gamma_{0}}\right\|_{L^{q_{0}\left(\Gamma_{0}\right)}} \leqslant C^{\varepsilon}\|v\|_{W^{1, q_{0}\left(\Sigma_{0}^{\varepsilon}\right)}}, \\
\left\|v_{\mid \Gamma}\right\|_{L^{1}(\Gamma)} \leqslant C\left\|v_{\mid \Gamma}\right\|_{L^{p}(\Gamma)} \leqslant C\|v\|_{W^{1, p}(\Omega)} .
\end{gathered}
$$


It follows

$$
\begin{aligned}
\|v\|_{L^{p}(\Omega)} & \leqslant C\left[\|\nabla v\|_{L^{p}(\Omega)^{N}}+C^{\varepsilon}\|v\|_{W^{1, q_{0}\left(\Sigma_{0}^{\varepsilon}\right)}}\right] \\
& \leqslant C\left[\|\nabla v\|_{L^{p}(\Omega)^{N}}+C^{\varepsilon}\|\nabla v\|_{L^{q_{0}\left(\Sigma_{0}^{\varepsilon}\right)^{N}}}\right], \\
\|v\|_{L^{q}\left(\Sigma^{\varepsilon}\right)} & \leqslant C^{\varepsilon}\left[\|\nabla v\|_{L^{q}\left(\Sigma^{\varepsilon}\right)^{N}}+\|v\|_{W^{1, p}(\Omega)}\right] \\
& \leqslant C^{\varepsilon}\left[\|\nabla v\|_{L^{q}\left(\Sigma^{\varepsilon}\right)^{N}}+\|\nabla v\|_{L^{p}(\Omega)^{N}}+\|\nabla v\|_{L^{q_{0}\left(\Sigma_{0}^{\varepsilon}\right)^{N}}}\right] .
\end{aligned}
$$

This gives the equivalence of the two norms under consideration.

Clearly $J^{\varepsilon}$ is a continuous strictly convex functional on $V^{\varepsilon}$. Moreover $J^{\varepsilon}$ is coercive since by (2.5), (2.7), (2.8) and the above equivalence, one has with $\alpha^{\varepsilon}$ such that $0<\alpha^{\varepsilon} \leqslant \min \left(\inf _{\sigma \in \Gamma} a^{\varepsilon}(\sigma) h^{\varepsilon}(\sigma)^{q-1}, \inf _{\sigma \in \Gamma_{0}} a_{0}^{\varepsilon}(\sigma) h_{0}^{\varepsilon}(\sigma)^{q_{0}-1}\right)$,

$$
\begin{aligned}
& J^{\varepsilon}(v) \geqslant \lambda\|\nabla v\|_{L^{p}(\Omega)^{N}}^{p}-\lambda^{\prime}\|v\|_{W^{1, p}(\Omega)} \\
& +\mu_{1} \alpha^{\varepsilon} \delta_{2}^{-q}\|\nabla v\|_{L^{q}\left(\Sigma^{\varepsilon}\right)^{N}}^{q}+\mu_{1} \alpha^{\varepsilon} \delta_{2}^{-q_{0}}\|\nabla v\|_{L^{q_{0}}\left(\Sigma_{0}^{\varepsilon}\right)^{N}}^{q_{0}} \\
& -\left\|f^{\varepsilon}\right\|_{L^{p^{\prime}}(\Omega)}\|v\|_{W^{1, p}(\Omega)}-\left\|g^{\varepsilon}\right\|_{L^{q^{\prime}\left(\Sigma^{\varepsilon}\right)}}\|v\|_{W^{1, q}\left(\Sigma^{\varepsilon}\right)} \\
& -\left\|g^{\varepsilon}\right\|_{L^{q_{0}^{\prime}\left(\Sigma_{0}^{\varepsilon}\right)}}\|v\|_{W^{1, q_{0}\left(\Sigma_{0}^{\varepsilon}\right)}}-C^{\varepsilon}\|v\|_{W^{1, q}\left(\Sigma^{\varepsilon}\right)} \\
& \geqslant \lambda\|\nabla v\|_{L^{p}(\Omega)^{N}}^{p}+\mu_{1} \alpha^{\varepsilon}\left[\delta_{2}^{-q}\|\nabla v\|_{L^{q}\left(\Sigma^{\varepsilon}\right)^{N}}^{q}+\delta_{2}^{-q_{0}}\|\nabla v\|_{L^{q_{0}\left(\Sigma_{0}^{\varepsilon}\right)^{N}}}^{q_{0}}\right] \\
& -C^{\varepsilon}\left[\|v\|_{W^{1, p}(\Omega)}+\|v\|_{W^{1, q}\left(\Sigma^{\varepsilon}\right)}+\|v\|_{W^{1, q_{0}}\left(\Sigma_{0}^{\varepsilon}\right)}\right] \\
& \geqslant\left[\lambda\|\nabla v\|_{L^{p}(\Omega)^{N}}^{p}-C^{\varepsilon}\|\nabla v\|_{L^{p}(\Omega)}\right] \\
& +\left[\mu_{1} \alpha^{\varepsilon} \delta_{2}^{-q}\|\nabla v\|_{L^{q}\left(\Sigma^{\varepsilon}\right)^{N}}^{q}-C^{\varepsilon}\|\nabla v\|_{L^{q}\left(\Sigma^{\varepsilon}\right)^{N}}\right] \\
& +\left[\mu_{1} \alpha^{\varepsilon} \delta_{2}^{-q_{0}}\|\nabla v\|_{L^{q_{0}}\left(\Sigma_{0}^{\varepsilon}\right)^{N}}^{q_{0}}-C^{\varepsilon}\|\nabla v\|_{L^{q_{0}}\left(\Sigma_{0}^{\varepsilon}\right)^{N}}\right]
\end{aligned}
$$

and since, when $\|v\|_{V^{\varepsilon}} \rightarrow+\infty$, at least one of $\|\nabla v\|_{L^{p}(\Omega)^{N}},\|\nabla v\|_{L^{q}\left(\Sigma^{\varepsilon}\right)^{N}}$ or $\|\nabla v\|_{L^{q_{0}}\left(\Sigma_{0}^{\varepsilon}\right)^{N}}$ tends to infinity.

We study the limit behaviour of $\left(\mathcal{P}^{\varepsilon}\right)$ under the following additional assumptions on $a^{\varepsilon}, a_{0}^{\varepsilon}, h^{\varepsilon}, h_{0}^{\varepsilon}, I^{\varepsilon}, f^{\varepsilon}, g^{\varepsilon}, g_{0}^{\varepsilon}$, valid when $\varepsilon$ tends to zero. First we assume that $\left\{\frac{1}{a^{\varepsilon}}\right\}_{\varepsilon}$ and $\left\{\frac{1}{a_{0}^{\varepsilon}}\right\}$ are respectively bounded in $L^{\infty}(\Gamma)$ and $L^{\infty}\left(\Gamma_{0}\right)$ :

$$
\exists \alpha>0 \text {, a.e. } s \in \Gamma \text {, a.e. } s_{0} \in \Gamma_{0}, \quad \forall \varepsilon, a^{\varepsilon}(s) \geqslant \alpha, a_{0}^{\varepsilon}\left(s_{0}\right) \geqslant \alpha .
$$

Moreover we assume that, up to a set of $(N-1)$-dimensional measure zero, there exists a partition of $\Gamma$ (respectively $\Gamma_{0}$ ) into two open regular subsets $\underline{\Gamma}$ and $\bar{\Gamma}$ (respectively $\underline{\Gamma}_{0}$ and $\bar{\Gamma}_{0}$ ) independent of $\varepsilon$ (one of them being possibly empty, none of them being necessarily connected), such that

$$
\begin{gathered}
\text { either } \underline{\Gamma}=\emptyset \quad \text { or } \quad \frac{1}{a^{\varepsilon} \mid \underline{\Gamma}} \rightarrow 0 \text { in } L^{q^{\prime}-1}(\underline{\Gamma}) \\
\text { (respectively either } \left.\underline{\Gamma}_{0}=\emptyset \text { or } \frac{1}{a_{0 \mid \underline{\Gamma}_{0}}^{\varepsilon}} \rightarrow 0 \text { in } L^{q_{0}^{\prime}-1}\left(\underline{\Gamma}_{0}\right)\right)
\end{gathered}
$$

and 


$$
\text { either } \bar{\Gamma}=\emptyset \quad \text { or } \quad \exists a \in L^{\infty}(\bar{\Gamma}), a_{\mid \bar{\Gamma}}^{\varepsilon} \rightarrow a \quad \text { in weak } \star-L^{\infty}(\bar{\Gamma})
$$

(respectively either $\bar{\Gamma}_{0}=\emptyset \quad$ or $\exists a_{0} \in L^{\infty}\left(\bar{\Gamma}_{0}\right), a_{0 \mid \bar{\Gamma}_{0}}^{\varepsilon} \rightarrow a_{0} \quad$ in weak $\left.\star-L^{\infty}\left(\bar{\Gamma}_{0}\right)\right)$,

$$
\nabla h^{\varepsilon} \rightarrow 0 \quad \text { in } L^{q}(\bar{\Gamma}) \quad\left(\text { respectively } \nabla h_{0}^{\varepsilon} \rightarrow 0 \quad \text { in } L^{q_{0}}\left(\bar{\Gamma}_{0}\right)\right) .
$$

We also assume that

for any $v \in K$ with $v_{\mid \underline{\Gamma}}=l$ (constant), $v_{\mid \underline{\Gamma}_{0}}=0, v_{\mid \bar{\Gamma}} \in L^{q}(\bar{\Gamma}), v_{\mid \bar{\Gamma}_{0}} \in L^{q_{0}}\left(\bar{\Gamma}_{0}\right)$, there exists a sequence of elements $v_{n} \in \mathcal{C}^{1}(\bar{\Omega}) \cap K$ such that $v_{n \mid \underline{\Gamma}}=l, v_{\mid \underline{\Gamma}_{0}}=0, v_{n} \rightarrow v$ in $W^{1, p}(\Omega), v_{n \mid \bar{\Gamma}} \rightarrow v_{\mid \bar{\Gamma}}$ in $L^{q}(\bar{\Gamma}), v_{n \mid \bar{\Gamma}_{0}} \rightarrow v_{\mid \bar{\Gamma}_{0}}$ in $L^{q_{0}}\left(\bar{\Gamma}_{0}\right)$.

Finally we assume that

$$
\begin{aligned}
& I^{\varepsilon} \rightarrow I,\left\{\left\|g^{\varepsilon}\right\|_{L^{q^{\prime}\left(\Sigma^{\varepsilon}\right)}}\right\}_{\varepsilon} \text { and }\left\{\left\|g_{0}^{\varepsilon}\right\|_{L^{q_{0}^{\prime}\left(\Sigma_{0}^{\varepsilon}\right)}}\right\}_{\varepsilon} \text { are bounded and } \\
& \exists f \in L^{p^{\prime}}(\Omega), f^{\varepsilon} \rightarrow f \text { weakly in } L^{p^{\prime}}(\Omega) .
\end{aligned}
$$

Let us comment (2.12). It means that the oscillations of $h^{\varepsilon}$ and $h_{0}^{\varepsilon}$ on $\bar{\Gamma}$ and $\bar{\Gamma}_{0}$ are small. Of course this holds true if (e.g.) $h^{\varepsilon}(s) \equiv \varepsilon h(s)$ with $h$ of class $\mathcal{C}^{1}(\partial \Omega), 0<h \leqslant 1$, but also if (e.g.) $N=2, \Gamma$ is a closed curve, $\varepsilon=n^{-r}, h^{\varepsilon}(s)=n^{-r} \mathcal{H}(n y(s))$ for $s \in \Gamma$, $y(s)=\operatorname{arc~length}(s), \mathcal{H}$ periodic of period $Y$ where $Y$ is the length of $\Gamma, 0<\mathcal{H} \leqslant 1, \mathcal{H}$ of class $\mathcal{C}^{1}\left(\mathbb{R}^{+}\right)$and $r>1$. (Actually in this case $\left|\nabla h^{\varepsilon}\right| \rightarrow 0$ in $L^{\infty}(\bar{\Gamma})$.) On the contrary it does not hold true in the periodic case of [7] or [11], where $r=1$, unless if, in the notations of [7], $R^{\varepsilon} / S^{\varepsilon}$ tends to zero.

Under the above assumptions we have

THEOREM 1. - Let $u^{\varepsilon}$ be the solution of

$$
\operatorname{Inf}\left\{J^{\varepsilon}(v), v \in V^{\varepsilon}, v_{\mid \Omega} \in K\right\}
$$

where $K$ is a nonempty closed convex subset of $W^{1, p}(\Omega)$, corresponding to conditions that concern neither $\Gamma$ nor $\Gamma_{0}$,

$$
\begin{gathered}
V^{\varepsilon}=\left\{v: \Omega^{\varepsilon} \rightarrow \mathbb{R}, v_{\mid \Omega} \in W^{1, p}(\Omega), v_{\mid \Sigma^{\varepsilon}} \in W^{1, q}\left(\Sigma^{\varepsilon}\right), v_{\mid \Sigma_{0}^{\varepsilon}} \in W^{1, q_{0}}\left(\Sigma_{0}^{\varepsilon}\right),\right. \\
\left.v_{\mid \Omega}=v_{\mid \Sigma^{\varepsilon}} \text { on } \Gamma, v_{\mid \Omega}=v_{\mid \Sigma_{0}^{\varepsilon}} \text { on } \Gamma_{0}, v_{\mid \Gamma^{\varepsilon}} \text { is constant }, v_{\mid \Gamma_{0}^{\varepsilon}}=0\right\}, \\
J^{\varepsilon}(v)=F\left(v_{\mid \Omega}\right)+\int_{\Sigma^{\varepsilon}} \frac{a^{\varepsilon} \circ \sigma}{h^{\varepsilon} \circ \sigma} G\left(h^{\varepsilon} \circ \sigma H^{o}(\nabla v)\right) \mathrm{d} x+\int_{\Sigma_{0}^{\varepsilon}} \frac{a_{0}^{\varepsilon} \circ \sigma_{0}}{h_{0}^{\varepsilon} \circ \sigma_{0}} G_{0}\left(h_{0}^{\varepsilon} \circ \sigma_{0} H_{0}^{o}(\nabla v)\right) \mathrm{d} x \\
-\int_{\Omega} f^{\varepsilon} v \mathrm{~d} x-\int_{\Sigma^{\varepsilon}} g^{\varepsilon} v \mathrm{~d} x-\int_{\Sigma_{0}^{\varepsilon}} g_{0}^{\varepsilon} v \mathrm{~d} x-I^{\varepsilon} v_{\mid \Gamma^{\varepsilon}} .
\end{gathered}
$$

Let us define $(\mathcal{P})$ by

$$
\operatorname{Inf}\{J(v, l) ;(v, l) \in W, v \in K\},
$$




$$
\begin{aligned}
W=\left\{(v, l) \in W^{1, p}(\Omega) \times \mathbb{R}, v_{\mid \bar{\Gamma}} \in L^{q}(\bar{\Gamma}), v_{\mid \bar{\Gamma}_{0}} \in L^{q_{0}}\left(\bar{\Gamma}_{0}\right),\right. \\
\left.v_{\mid \underline{\Gamma}}=l(\text { if } \underline{\Gamma} \neq \emptyset), v_{\mid \underline{\Gamma}_{0}}=0\left(\text { if } \underline{\Gamma}_{0} \neq \emptyset\right)\right\}
\end{aligned}
$$

(the restriction $v_{\mid \bar{\Gamma}} \in L^{q}(\bar{\Gamma})$ being effective only if $\bar{\Gamma} \neq \emptyset$ and $q>p$, similarly for $\bar{\Gamma}_{0}$ ),

$$
J(v, l)=F(v)+\int_{\bar{\Gamma}} a G(|v-l|) H^{o}(n) \mathrm{d} s+\int_{\bar{\Gamma}_{0}} a_{0} G_{0}(|v|) H_{0}^{o}(n) \mathrm{d} s-\int_{\Omega} f v \mathrm{~d} x-I l,
$$

where $n=n(s)$ is the unit normal to $\bar{\Gamma}$ or $\bar{\Gamma}_{0}$ at the point $s$.

Then $(\mathcal{P})$ has a unique solution $(u, k) \in W$ with $u \in K$. Moreover when $\varepsilon \rightarrow 0$,

(1) $u^{\varepsilon}{ }_{\mid \Omega}$ tends to $u$ in weak- $W^{1, p}(\Omega)$ and in $L^{p}(\Omega)$;

(2) the function $\tilde{v}^{\varepsilon}: \Sigma^{\prime} \rightarrow \mathbb{R}$ given by $\tilde{v}^{\varepsilon}=u^{\varepsilon}-u_{\mid \Gamma^{\varepsilon}}^{\varepsilon}$ in $\Sigma^{\varepsilon}, 0$ in $\Sigma^{\prime} \backslash \Sigma^{\varepsilon}$ tends to zero in $L^{q}\left(\Sigma^{\prime}\right)$; the function $\tilde{v}_{0}^{\varepsilon}: \Sigma_{0}^{\prime} \rightarrow \mathbb{R}$ given by $\tilde{v}_{0}^{\varepsilon}=u^{\varepsilon}$ in $\Sigma_{0}^{\varepsilon}, 0$ in $\Sigma_{0}^{\prime} \backslash \Sigma_{0}^{\varepsilon}$ tends to zero in $L^{q_{0}}\left(\Sigma_{0}^{\prime}\right)$;

(3) $u^{\varepsilon} \mid \Gamma \rightarrow u_{\mid \Gamma}$ in $L^{p}(\Gamma), u_{\mid \Gamma}^{\varepsilon} \rightarrow u_{\mid \Gamma}$ in weak- $L^{q}(\Gamma), u^{\varepsilon}{ }_{\mid \Gamma_{0}} \rightarrow u_{\mid \Gamma_{0}}$ in $L^{p}\left(\Gamma_{0}\right), u^{\varepsilon} \mid \Gamma_{0} \rightarrow$ $u_{\mid \Gamma_{0}}$ in weak- $L^{q_{0}}\left(\Gamma_{0}\right)$, the weak convergences being of interest only if $q>p$ (respectively $\left.\left.q_{0}>p\right)\right), u_{\mid \Gamma^{\varepsilon}}^{\varepsilon} \rightarrow k$ in $\mathbb{R}$;

(4) $J^{\varepsilon}\left(u^{\varepsilon}\right) \rightarrow J(u, k)$.

Except for an example given in the last section, the rest of the paper is devoted to the proof of this theorem.

\section{Existence and uniqueness of the solution $(u, k)$ of $(\mathcal{P})$}

- If $\bar{\Gamma}=\emptyset$, that is if $\underline{\Gamma}=\Gamma,(\mathcal{P})$ reduces to

$$
\operatorname{Inf}\left\{J^{\prime}(v), v \in V^{\prime} \cap K\right\},
$$

where

$$
\begin{gathered}
V^{\prime}=\left\{v \in W^{1, p}(\Omega), v_{\mid \Gamma} \text { is constant }, v_{\mid \bar{\Gamma}_{0}} \in L^{q_{0}}\left(\bar{\Gamma}_{0}\right), v_{\mid \Sigma_{0}}=0\right\}, \\
J^{\prime}(v)=F(v)+\int_{\bar{\Gamma}_{0}} a_{0} G_{0}(|v|) H_{0}^{o}(n) \mathrm{d} s-\int_{\Omega} f v \mathrm{~d} x-I v_{\mid \Gamma} .
\end{gathered}
$$

If also $\bar{\Gamma}_{0}$ (together with $\bar{\Gamma}$ ) is empty, then $V^{\prime}$ reduces to

$$
\left\{v \in W^{1, p}(\Omega), v_{\mid \Gamma_{0}}=0, v_{\mid \Gamma} \text { is constant }\right\},
$$

$J^{\prime}(v)$ reduces to

$$
F(v)-\int_{\Omega} f v \mathrm{~d} x-I v_{\mid \Gamma}
$$

and it is very classical that $\left(\mathcal{P}^{\prime}\right)$ admits a unique solution $u \in V^{\prime} \cap K$. If $\bar{\Gamma}=\emptyset$ and $\bar{\Gamma}_{0} \neq \emptyset$, then $V^{\prime}$ is a Banach space for

$$
\|v\|_{V^{\prime}}=\|\nabla v\|_{L^{p}(\Omega)^{N}}+\|v\|_{L^{q_{0}}\left(\bar{\Gamma}_{0}\right)}
$$


and for any $v \in V^{\prime}$, one has by Poincaré inequality

$$
\|v\|_{L^{p}(\Omega)} \leqslant C\|\nabla v\|_{L^{p}(\Omega)^{N}}+C\|v\|_{L^{1}\left(\bar{\Gamma}_{0}\right)} \leqslant C\|\nabla v\|_{L^{p}(\Omega)^{N}}+C\|v\|_{L^{q_{0}}\left(\bar{\Gamma}_{0}\right)},
$$

so that $J^{\prime}$ is coercive on $V^{\prime}$.

- Otherwise, that is if $\bar{\Gamma} \neq \emptyset, W$ is a Banach space for

$$
\|(v, l)\|_{W}=\|\nabla v\|_{L^{p}(\Omega)^{N}}+\|v\|_{L^{q_{0}}\left(\bar{\Gamma}_{0}\right)}+\|v-l\|_{L^{q}(\bar{\Gamma})} .
$$

Note that this norm is equivalent to the natural one:

$$
\||(v, l)|\|=\|v\|_{W^{1, p}(\Omega)}+\|v\|_{L^{q_{0}\left(\bar{\Gamma}_{0}\right)}}+\|v\|_{L^{q}(\bar{\Gamma})}+|l| .
$$

Actually since

$$
\|v-l\|_{L^{q}(\bar{\Gamma})} \leqslant\|v\|_{L^{q}(\bar{\Gamma})}+\|l\|_{L^{q}(\bar{\Gamma})} \leqslant\|v\|_{L^{q}(\bar{\Gamma})}+C|l|,
$$

we get clearly $\|(v, l)\|_{W} \leqslant\||(v, l) \||$; on the contrary by Poincaré inequality,

$$
\begin{aligned}
\|v\|_{W^{1, p}(\Omega)} & \leqslant C\|\nabla v\|_{L^{p}(\Omega)^{N}}+C\|v\|_{L^{1}\left(\bar{\Gamma}_{0}\right)} \\
& \leqslant C\|\nabla v\|_{L^{p}(\Omega)^{N}}+C\|v\|_{L^{q_{0}}\left(\bar{\Gamma}_{0}\right)} \leqslant C\|(v, l)\|_{W}, \\
|l| & =\frac{1}{|\bar{\Gamma}|}\left|\int_{\bar{\Gamma}} v \mathrm{~d} s-\int_{\bar{\Gamma}}(v-l) \mathrm{d} s\right| \leqslant C \int_{\bar{\Gamma}}|v| \mathrm{d} s+C \int_{\bar{\Gamma}}|v-l| \mathrm{d} s \\
& \leqslant C\|v\|_{W^{1, p}(\Omega)}+C\|v-l\|_{L^{q}(\bar{\Gamma})} \leqslant C\|(v, l)\|_{W}, \\
\|v\|_{L^{q}(\bar{\Gamma})} & \leqslant\|v-l\|_{L^{q}(\bar{\Gamma})}+C|l| \leqslant\|v-l\|_{L^{q}(\bar{\Gamma})}+C\|(v, l)\|_{W} \leqslant C\|(v, l)\|_{W}
\end{aligned}
$$

and we get $\left\|\left|(v, l)\|\mid \leqslant C\|(v, l) \|_{W}\right.\right.$. The functional $J$ is strictly convex and continuous on $W$ and it is coercive since by $(2.5),(2.7),(2.8),(2.9),(2.11),(2.14)$ and by the above inequalities

$$
\begin{aligned}
J(v, l) \geqslant & \lambda\|\nabla v\|_{L^{p}(\Omega)^{N}}^{p}-\lambda^{\prime}\|v\|_{W^{1, p}(\Omega)}+\alpha \mu_{1} \delta_{2}^{-1}\left[\|v-l\|_{L^{q}(\bar{\Gamma})}^{q}+\|v\|_{L^{q_{0}\left(\bar{\Gamma}_{0}\right)}}^{q_{0}}\right] \\
& -\|f\|_{L^{p^{\prime}(\Omega)}}\|v\|_{W^{1, p}(\Omega)}-|I|\left[C\|v\|_{W^{1, p}(\Omega)}+C\|v-l\|_{L^{q}(\bar{\Gamma})}\right] \\
\geqslant & \lambda\|\nabla v\|_{L^{p}(\Omega)^{N}}^{p}-C\|\nabla v\|_{L^{p}(\Omega)^{N}}+\left[\alpha \mu_{1} \delta_{2}^{-1}\|v-l\|_{L^{q}(\bar{\Gamma})}^{q}-C\|v-l\|_{L^{q}(\bar{\Gamma})}\right] \\
& +\left[\alpha \mu_{1} \delta_{2}^{-1}\|v\|_{L^{q_{0}\left(\bar{\Gamma}_{0}\right)}}^{q_{0}}-C\|v\|_{L^{q_{0}}\left(\bar{\Gamma}_{0}\right)}\right]
\end{aligned}
$$

and since, when $\|(v, l)\|_{W} \rightarrow+\infty$, either $\|\nabla v\|_{L^{p}(\Omega)^{N}}$ or $\|v\|_{L^{q_{0}}\left(\bar{\Gamma}_{0}\right)}$, or $\|v-l\|_{L^{q}(\bar{\Gamma})}$ tends to infinity.

Remark 1. - Note that if $\underline{\Gamma}=\emptyset$, there is no link between $v$ and $l$ for $(v, l)$ in $W$.

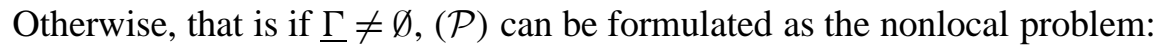

$$
\operatorname{Inf}\left\{J^{\prime \prime}(v), v \in V^{\prime \prime} \cap K\right\},
$$

with

$$
V^{\prime \prime}=\left\{v \in W^{1, p}(\Omega), v_{\mid \underline{\Gamma}_{0}}=0, v_{\mid \bar{\Gamma}_{0}} \in L^{q_{0}}\left(\bar{\Gamma}_{0}\right), v_{\mid \bar{\Gamma}} \in L^{q}(\bar{\Gamma}), v_{\mid \underline{\Gamma}} \text { is constant }\right\}
$$




$$
J^{\prime \prime}(v)=F(v)+\int_{\frac{\Gamma}{\Gamma}} a G\left(\left|v-v_{\mid \underline{\Gamma}}\right|\right) H^{o}(n) \mathrm{d} s+\int_{\bar{\Gamma}_{0}} a_{0} G_{0}(|v|) H_{0}^{o}(n) \mathrm{d} s-\int_{\Omega} f v \mathrm{~d} x-I v_{\mid \underline{\Gamma}} .
$$

\section{A priori estimates and consequences for $u^{\varepsilon}$}

The a priori estimates are given in

LEMMA 1. - (1) $\exists C>0, \forall \varepsilon, \forall v \in W^{1, q}\left(\Sigma^{\varepsilon}\right), v_{\mid \Gamma^{\varepsilon}}=k^{\varepsilon}$ (constant) $\Rightarrow$

$$
\begin{aligned}
& \int_{\Sigma^{\varepsilon}}\left|v-k^{\varepsilon}\right|^{q} \mathrm{~d} x \leqslant C \int_{\Sigma^{\varepsilon}}\left(h^{\varepsilon} \circ \sigma\right)^{q} H^{o}(\nabla v)^{q} \mathrm{~d} x, \\
& \int_{\Gamma}\left|v-k^{\varepsilon}\right|^{q} \mathrm{~d} s \leqslant C \int_{\Sigma^{\varepsilon}}\left(h^{\varepsilon} \circ \sigma\right)^{q-1} H^{o}(\nabla v)^{q} \mathrm{~d} x,
\end{aligned}
$$

which applies to $u^{\varepsilon}$, giving also

$$
\begin{aligned}
& \int_{\Sigma_{0}^{\varepsilon}}\left|u^{\varepsilon}\right|^{q_{0}} \mathrm{~d} x \leqslant C \int_{\Sigma_{0}^{\varepsilon}}\left(h_{0}^{\varepsilon} \circ \sigma_{0}\right)^{q_{0}} H_{0}^{o}\left(\nabla u^{\varepsilon}\right)^{q_{0}} \mathrm{~d} x, \\
& \int_{\Gamma_{0}}\left|u^{\varepsilon}\right|^{q_{0}} \mathrm{~d} s \leqslant C \int_{\Sigma_{0}^{\varepsilon}}\left(h_{0}^{\varepsilon} \circ \sigma_{0}\right)^{q_{0}-1} H_{0}^{o}\left(\nabla u^{\varepsilon}\right)^{q_{0}} \mathrm{~d} x .
\end{aligned}
$$

(2) $u^{\varepsilon}{ }_{\mid \Omega}$ is bounded in $W^{1, p}(\Omega), u^{\varepsilon} \Gamma^{\varepsilon}$ is bounded in $\mathbb{R}$.

(3) $F\left(u^{\varepsilon} \mid \Omega\right), \int_{\Sigma^{\varepsilon}} \frac{a^{\varepsilon} \circ \sigma}{h^{\varepsilon} \circ \sigma} G\left(h^{\varepsilon} \circ \sigma H^{o}\left(\nabla u^{\varepsilon}\right)\right) \mathrm{d} x$ and $\int_{\Sigma_{0}^{\varepsilon}} \frac{a_{0}^{\varepsilon} \circ \sigma_{0}}{h_{0}^{\varepsilon} \circ \sigma_{0}} G\left(h_{0}^{\varepsilon} \circ \sigma H_{0}^{o}\left(\nabla u^{\varepsilon}\right)\right) \mathrm{d} x$ are bounded.

(4) $u^{\varepsilon}{ }_{\mid \Gamma}$ is bounded in $L^{q}(\Gamma)$ and $u^{\varepsilon}{ }_{\mid \Gamma_{0}}$ is bounded in $L^{q_{0}}\left(\Gamma_{0}\right)$.

(5) $\int_{\Sigma^{\varepsilon}}\left|u^{\varepsilon}-u_{\mid \Gamma^{\varepsilon}}^{\varepsilon}\right|^{q} \mathrm{~d} x$ and $\int_{\Sigma_{0}^{\varepsilon}}\left|u^{\varepsilon}\right|^{q_{0}} \mathrm{~d} x$ tend to zero.

Proof of (1). - By using the $\mathcal{C}^{1}$-diffeomorphism of $\Sigma_{0}^{\prime}$ onto $\Gamma_{0} \times\left(0, t^{\prime}\right)$, it is proved in [5] that $\exists C>0, \forall \varepsilon, \forall w \in W^{1, q_{0}}\left(\Sigma_{0}^{\varepsilon}\right), w_{\mid \Gamma_{0}^{\varepsilon}}=0 \Rightarrow$

$$
\begin{aligned}
& \int_{\Sigma_{0}^{\varepsilon}}|w|^{q_{0}} \mathrm{~d} x \leqslant C \int_{\Sigma_{0}^{\varepsilon}}\left(h_{0}^{\varepsilon} \circ \sigma_{0}\right)^{q_{0}} H_{0}^{o}(\nabla w)^{q_{0}} \mathrm{~d} x, \\
& \int_{\Gamma_{0}}|w|^{q_{0}} \mathrm{~d} s \leqslant C \int_{\Sigma_{0}^{\varepsilon}}\left(h_{0}^{\varepsilon} \circ \sigma_{0}\right)^{q_{0}-1} H_{0}^{o}(\nabla w)^{q_{0}} \mathrm{~d} x .
\end{aligned}
$$

Applying this to $w=v-k^{\varepsilon}$ and deleting the subscript zero gives the first two estimates.

Proof of (2), (3) and (4). - Let $k^{\varepsilon}=u_{\mid \Gamma^{\varepsilon}}^{\varepsilon}$. It follows from (1) that

$$
\left\|u^{\varepsilon}-k^{\varepsilon}\right\|_{L^{1}(\Gamma)} \leqslant C\left\|u^{\varepsilon}-k^{\varepsilon}\right\|_{L^{q}(\Gamma)} \leqslant C\left[\int_{\Sigma^{\varepsilon}}\left(h^{\varepsilon} \circ \sigma\right)^{q-1} H^{o}\left(\nabla u^{\varepsilon}\right)^{q} \mathrm{~d} x\right]^{1 / q}
$$

and hence 


$$
\begin{aligned}
C\left|k^{\varepsilon}\right|=\left\|k^{\varepsilon}\right\|_{L^{1}(\Gamma)} \leqslant & \left\|u^{\varepsilon}\right\|_{L^{1}(\Gamma)}+C\left[\int_{\Sigma^{\varepsilon}}\left(h^{\varepsilon} \circ \sigma\right)^{q-1} H^{o}\left(\nabla u^{\varepsilon}\right)^{q} \mathrm{~d} x\right]^{1 / q} \\
\leqslant & \left\|u^{\varepsilon}\right\|_{W^{1, p}(\Omega)}+C\left[\int_{\Sigma^{\varepsilon}}\left(h^{\varepsilon} \circ \sigma\right)^{q-1} H^{o}\left(\nabla u^{\varepsilon}\right)^{q} \mathrm{~d} x\right]^{1 / q} \\
\leqslant & \left\|\nabla u^{\varepsilon}\right\|_{L^{p}(\Omega)^{N}}+C\left\|u^{\varepsilon}\right\|_{L^{q_{0}\left(\Gamma_{0}\right)}} \\
& +C\left[\int_{\Sigma^{\varepsilon}}\left(h^{\varepsilon} \circ \sigma\right)^{q-1} H^{o}\left(\nabla u^{\varepsilon}\right)^{q} \mathrm{~d} x\right]^{1 / q}
\end{aligned}
$$

and it follows that

$$
\begin{aligned}
\left|k^{\varepsilon}\right| \leqslant & C\left\|\nabla u^{\varepsilon}\right\|_{L^{p}(\Omega)^{N}}+C\left[\int_{\Sigma_{0}^{\varepsilon}}\left(h_{0}^{\varepsilon} \circ \sigma_{0}\right)^{q_{0}-1} H_{0}^{o}\left(\nabla u^{\varepsilon}\right)^{q_{0}} \mathrm{~d} x\right]^{1 / q_{0}} \\
& +C\left[\int_{\Sigma^{\varepsilon}}\left(h^{\varepsilon} \circ \sigma\right)^{q-1} H^{o}\left(\nabla u^{\varepsilon}\right)^{q} \mathrm{~d} x\right]^{1 / q} .
\end{aligned}
$$

Moreover for small $\varepsilon(\varepsilon \leqslant 1)$, we have using (1) again

$$
\begin{aligned}
\left\|u^{\varepsilon}\right\|_{L^{q}\left(\Sigma^{\varepsilon}\right)} & \leqslant C\left\|k^{\varepsilon}\right\|_{L^{q}\left(\Sigma^{\varepsilon}\right)}+C\left[\int_{\Sigma^{\varepsilon}}\left(h^{\varepsilon} \circ \sigma\right)^{q} H^{o}\left(\nabla u^{\varepsilon}\right)^{q} \mathrm{~d} x\right]^{1 / q} \\
& \leqslant C\left|k^{\varepsilon}\right|+C\left[\int_{\Sigma^{\varepsilon}}\left(h^{\varepsilon} \circ \sigma\right)^{q} H^{o}\left(\nabla u^{\varepsilon}\right)^{q} \mathrm{~d} x\right]^{1 / q}
\end{aligned}
$$

and by means of (4.1)

$$
\begin{aligned}
\left\|u^{\varepsilon}\right\|_{L^{q}\left(\Sigma^{\varepsilon}\right)} \leqslant & C\left\|\nabla u^{\varepsilon}\right\|_{L^{p}(\Omega)^{N}}+C\left[\int_{\Sigma^{\varepsilon}}\left(h^{\varepsilon} \circ \sigma\right)^{q-1} H^{o}\left(\nabla u^{\varepsilon}\right)^{q} \mathrm{~d} x\right]^{1 / q} \\
& +C\left[\int_{\Sigma_{0}^{\varepsilon}}\left(h_{0}^{\varepsilon} \circ \sigma_{0}\right)^{q_{0}-1} H_{0}^{o}\left(\nabla u_{0}^{\varepsilon}\right)^{q_{0}} \mathrm{~d} x\right]^{1 / q_{0}} .
\end{aligned}
$$

Similarly one has by making $k^{\varepsilon}=0$ in the penultimate inequality

$$
\left\|u^{\varepsilon}\right\|_{L^{q_{0}}\left(\Sigma_{0}^{\varepsilon}\right)} \leqslant C\left[\int_{\Sigma_{0}^{\varepsilon}}\left(h_{0}^{\varepsilon} \circ \sigma_{0}\right)^{q_{0}} H_{0}^{o}\left(\nabla u^{\varepsilon}\right)^{q_{0}} \mathrm{~d} x\right]^{1 / q_{0}} .
$$

Now let $\alpha$ be as in (2.9). One has by (2.8)

$$
\begin{aligned}
\alpha \int_{\Sigma^{\varepsilon}}\left(h^{\varepsilon} \circ \sigma\right)^{q-1} H^{o}\left(\nabla u^{\varepsilon}\right)^{q} \mathrm{~d} x & \leqslant \int_{\Sigma^{\varepsilon}}\left(a^{\varepsilon} \circ \sigma\right)\left(h^{\varepsilon} \circ \sigma\right)^{q-1} H^{o}\left(\nabla u^{\varepsilon}\right)^{q} \mathrm{~d} x \\
& \leqslant \frac{1}{\mu_{1}} \int_{\Sigma^{\varepsilon}} \frac{a^{\varepsilon} \circ \sigma}{h^{\varepsilon} \circ \sigma} G\left(h^{\varepsilon} \circ \sigma H^{o}\left(\nabla u^{\varepsilon}\right)\right) \mathrm{d} x
\end{aligned}
$$


and similarly

$$
\alpha \int_{\Sigma_{0}^{\varepsilon}}\left(h_{0}^{\varepsilon} \circ \sigma_{0}\right)^{q_{0}-1} H_{0}^{o}\left(\nabla u^{\varepsilon}\right)^{q_{0}} \mathrm{~d} x \leqslant \frac{1}{\mu_{1}} \int_{\Sigma_{0}^{\varepsilon}} \frac{a_{0}^{\varepsilon} \circ \sigma_{0}}{h_{0}^{\varepsilon} \circ \sigma_{0}} G_{0}\left(h_{0}^{\varepsilon} \circ \sigma_{0} H_{0}^{o}\left(\nabla u^{\varepsilon}\right)\right) \mathrm{d} x
$$

So that by (2.7), (2.14), (4.1) to (4.3), one has with $v \in V^{\varepsilon}, v_{\mid \Omega} \in K, v=0$ in $\Sigma^{\varepsilon}$ and $\Sigma_{0}^{\varepsilon}$,

$$
\begin{aligned}
& \lambda\left\|\nabla u^{\varepsilon}\right\|_{L^{p}(\Omega)^{N}}^{p}-\lambda^{\prime}\left\|u^{\varepsilon}\right\|_{W^{1, p}(\Omega)} \\
& \quad+\mu_{1} \alpha \int_{\Sigma^{\varepsilon}}\left(h^{\varepsilon} \circ \sigma\right)^{q-1} H^{o}\left(\nabla u^{\varepsilon}\right)^{q} \mathrm{~d} x+\mu_{1} \alpha \int_{\Sigma_{0}^{\varepsilon}}\left(h_{0}^{\varepsilon} \circ \sigma_{0}\right)^{q_{0}-1} H_{0}^{o}\left(\nabla u^{\varepsilon}\right)^{q_{0}} \mathrm{~d} x \\
& \leqslant F\left(u_{\mid \Omega}^{\varepsilon}\right)+\int_{\Sigma^{\varepsilon}} \frac{a^{\varepsilon} \circ \sigma}{h^{\varepsilon} \circ \sigma} G\left(h^{\varepsilon} \circ \sigma H^{o}\left(\nabla u^{\varepsilon}\right)\right) \mathrm{d} x \\
& \quad+\int_{\Sigma_{0}^{\varepsilon}} \frac{a_{0}^{\varepsilon} \circ \sigma_{0}}{h_{0}^{\varepsilon} \circ \sigma_{0}} G_{0}\left(h_{0}^{\varepsilon} \circ \sigma_{0} H_{0}^{o}\left(\nabla u^{\varepsilon}\right)\right) \mathrm{d} x \\
& \leqslant F\left(v_{\mid \Omega}\right)+\int_{\Omega} f^{\varepsilon} u^{\varepsilon} \mathrm{d} x-\int_{\Omega} f^{\varepsilon} v d x+\int_{\Sigma^{\varepsilon}} g^{\varepsilon} u^{\varepsilon} \mathrm{d} x+\int_{\Sigma_{0}^{\varepsilon}} g_{0}^{\varepsilon} u^{\varepsilon} \mathrm{d} x+I^{\varepsilon} k^{\varepsilon}
\end{aligned}
$$

(as $u^{\varepsilon}$ solves $\left(\mathcal{P}^{\varepsilon}\right)$ and as $H^{o}(0)=H_{0}^{o}(0)=0$ and $G(0)=G_{0}(0)=0$ )

$$
\begin{aligned}
& \leqslant C+C\left\|u^{\varepsilon}\right\|_{W^{1, p}(\Omega)}+C\left\|u^{\varepsilon}\right\|_{L^{q}\left(\Sigma^{\varepsilon}\right)}+C\left\|u^{\varepsilon}\right\|_{L^{q_{0}\left(\Sigma_{0}^{\varepsilon}\right)}}+C\left|k^{\varepsilon}\right| \\
& \leqslant C+C\left\|u^{\varepsilon}\right\|_{W^{1, p}(\Omega)}+C\left(\int_{\Sigma^{\varepsilon}}\left(h^{\varepsilon} \circ \sigma\right)^{q-1} H^{o}\left(\nabla u^{\varepsilon}\right)^{q} \mathrm{~d} x\right)^{1 / q}
\end{aligned}
$$$$
+C\left(\int_{\Sigma_{0}^{\varepsilon}}\left(h_{0}^{\varepsilon} \circ \sigma_{0}\right)^{q_{0}-1} H_{0}^{o}\left(\nabla u^{\varepsilon}\right)^{q_{0}} \mathrm{~d} x\right)^{1 / q_{0}} .
$$

As already noticed

$$
\left\|u^{\varepsilon}\right\|_{W^{1, p}(\Omega)} \leqslant C\left\|\nabla u^{\varepsilon}\right\|_{L^{p}(\Omega)^{N}}+C\left(\int_{\Sigma_{0}^{\varepsilon}}\left(h_{0}^{\varepsilon} \circ \sigma_{0}\right)^{q_{0}-1} H_{0}^{o}\left(\nabla u^{\varepsilon}\right)^{q_{0}} \mathrm{~d} x\right)^{1 / q_{0}}
$$

and it follows from (4.6) and (4.7) that

$$
\begin{aligned}
& \mu_{1} \alpha \int_{\Sigma^{\varepsilon}}\left(h^{\varepsilon} \circ \sigma\right)^{q-1} H^{o}\left(\nabla u^{\varepsilon}\right)^{q} \mathrm{~d} x-C\left(\int_{\Sigma^{\varepsilon}}\left(h^{\varepsilon} \circ \sigma\right)^{q-1} H^{o}\left(\nabla u^{\varepsilon}\right)^{q} \mathrm{~d} x\right)^{1 / q} \\
& \quad+\mu_{1} \alpha \int_{\Sigma_{0}^{\varepsilon}}\left(h_{0}^{\varepsilon} \circ \sigma_{0}\right)^{q_{0}-1} H_{0}^{o}\left(\nabla u^{\varepsilon}\right)^{q_{0}} \mathrm{~d} x-C\left(\int_{\Sigma_{0}^{\varepsilon}}\left(h_{0}^{\varepsilon} \circ \sigma_{0}\right)^{q_{0}-1} H_{0}^{o}\left(\nabla u^{\varepsilon}\right)^{q_{0}} \mathrm{~d} x\right)^{1 / q_{0}} \\
& \quad+\lambda\left\|\nabla u^{\varepsilon}\right\|_{L^{p}(\Omega)^{N}}^{p}-C\left\|\nabla u^{\varepsilon}\right\|_{L^{p}(\Omega)^{N}} \leqslant C .
\end{aligned}
$$


We remark that each of the above lines has the form $C t^{p}-C^{\prime} t$, hence it is bounded below and tends to infinity with $t$. We conclude that

$$
\left\|\nabla u^{\varepsilon}\right\|_{L^{p}(\Omega)^{N}}, \quad \int_{\Sigma^{\varepsilon}}\left(h^{\varepsilon} \circ \sigma\right)^{q-1} H^{o}\left(\nabla u^{\varepsilon}\right)^{q} \mathrm{~d} x \quad \text { and } \quad \int_{\Sigma_{0}^{\varepsilon}}\left(h_{0}^{\varepsilon} \circ \sigma_{0}\right)^{q_{0}-1} H_{0}^{o}\left(\nabla u^{\varepsilon}\right)^{q_{0}} \mathrm{~d} x
$$

are bounded. By (4.7), $u^{\varepsilon}$ is bounded in $W^{1, p}(\Omega)$ and by (4.1), $k^{\varepsilon}=u_{\mid \Gamma^{\varepsilon}}^{\varepsilon}$ is bounded in $\mathbb{R}$. From the arguments following (4.5),

$F\left(u^{\varepsilon}{ }_{\mid \Omega}\right), \quad \int_{\Sigma^{\varepsilon}} \frac{a^{\varepsilon} \circ \sigma}{h^{\varepsilon} \circ \sigma} G\left(h^{\varepsilon} \circ \sigma H^{o}\left(\nabla u^{\varepsilon}\right)\right) \mathrm{d} x \quad$ and $\quad \int_{\Sigma_{0}^{\varepsilon}} \frac{a_{0}^{\varepsilon} \circ \sigma_{0}}{h_{0}^{\varepsilon} \circ \sigma_{0}} G_{0}\left(h_{0}^{\varepsilon} \circ \sigma_{0} H_{0}^{o}\left(\nabla u^{\varepsilon}\right)\right) \mathrm{d} x$

are bounded. By (1-) it is clear that $u^{\varepsilon}{ }_{\mid \Gamma_{0}}$ is bounded in $L^{q_{0}}\left(\Gamma_{0}\right)$. Moreover we get that $u_{\mid \Gamma}^{\varepsilon}-k^{\varepsilon}$ is bounded in $L^{q}(\Gamma)$ and as $k^{\varepsilon}$ is bounded, it follows that $u_{\mid \Gamma}^{\varepsilon}$ is bounded in $L^{q}(\Gamma)$.

Proof of (5). - From (1) and (2.6)

$\int_{\Sigma^{\varepsilon}}\left|u^{\varepsilon}-u_{\mid \Gamma^{\varepsilon}}^{\varepsilon}\right|^{q} \mathrm{~d} x \leqslant C \int_{\Sigma^{\varepsilon}}\left(h^{\varepsilon} \circ \sigma\right)^{q} H^{o}\left(\nabla u^{\varepsilon}\right)^{q} \mathrm{~d} x \leqslant C \varepsilon \int_{\Sigma^{\varepsilon}}\left(h^{\varepsilon} \circ \sigma\right)^{q-1} H^{o}\left(\nabla u^{\varepsilon}\right)^{q} \mathrm{~d} x \leqslant C \varepsilon$,

as we have just seen that $\int_{\Sigma^{\varepsilon}}\left(h^{\varepsilon} \circ \sigma\right)^{q-1} H^{o}\left(\nabla u^{\varepsilon}\right)^{q} \mathrm{~d} x$ is bounded. Similarly

$$
\int_{\Sigma_{0}^{\varepsilon}}\left|u^{\varepsilon}\right|^{q_{0}} \mathrm{~d} x \leqslant C \varepsilon
$$

From the a-priori estimates we are going to deduce

LEMMA 2. - There exists a subsequence $\varepsilon^{\prime}$ of $\varepsilon$ and an element $(u, k)$ of $W$ such that $u \in K$ and

$$
\begin{aligned}
& u^{\varepsilon^{\prime}}{ }_{\mid \Omega} \rightarrow u \quad \text { in weak- } W^{1, p}(\Omega), \quad u^{\varepsilon^{\prime}}{ }_{\mid \Omega} \rightarrow u \quad \text { in } L^{p}(\Omega), \\
& u^{\varepsilon^{\prime}}{ }_{\mid \Gamma^{\varepsilon^{\prime}}} \rightarrow k \quad(\text { in } \mathbb{R}) \text {, } \\
& \left.u^{\varepsilon^{\prime}}{ }_{\mid \Gamma} \rightarrow u_{\mid \Gamma} \quad \text { in } L^{p}(\Gamma) \quad \text { (hence in } L^{q}(\Gamma) \text { if } q \leqslant p\right) \text {, } \\
& \left.u^{\varepsilon^{\prime}}{ }_{\mid \Gamma_{0}} \rightarrow u_{\mid \Gamma_{0}} \text { in } L^{p}\left(\Gamma_{0}\right) \quad \text { (hence in } L^{q_{0}}\left(\Gamma_{0}\right) \text { if } q_{0} \leqslant p\right) \text {, } \\
& u^{\varepsilon^{\prime}}{ }_{\mid \Gamma} \rightarrow u_{\mid \Gamma} \quad \text { in weak }-L^{q}(\Gamma) \text {, } \\
& u^{\varepsilon^{\prime}}{ }_{\mid \Gamma_{0}} \rightarrow u_{\mid \Gamma_{0}} \text { in weak- } L^{q_{0}}\left(\Gamma_{0}\right),
\end{aligned}
$$

(and $\tilde{v}^{\varepsilon}=\left(u^{\varepsilon}-u_{\mid \Gamma^{\varepsilon}}^{\varepsilon}\right.$ in $\Sigma^{\varepsilon}, 0$ in $\left.\Sigma^{\prime} \backslash \Sigma^{\varepsilon}\right) \rightarrow 0$ in $L^{q}\left(\Sigma^{\prime}\right), \tilde{v}_{0}^{\varepsilon}=\left(u^{\varepsilon}\right.$ in $\Sigma_{0}^{\varepsilon}, 0$ in $\Sigma_{0}^{\prime} \backslash$ $\left.\Sigma_{0}^{\varepsilon}\right) \rightarrow 0$ in $\left.L^{q_{0}}\left(\Sigma_{0}^{\prime}\right)\right)$. Moreover

$$
\liminf \int_{\Sigma^{\varepsilon^{\prime}}} \frac{a^{\varepsilon^{\prime}} \circ \sigma}{h^{\varepsilon^{\prime}} \circ \sigma} G\left(h^{\varepsilon^{\prime}} \circ \sigma H^{o}\left(\nabla u^{\varepsilon^{\prime}}\right)\right) \mathrm{d} x \geqslant \int_{\bar{\Gamma}} a H^{o}(n) G(|u-k|) \mathrm{d} s
$$




$$
\liminf \int_{\Sigma_{0}^{\varepsilon^{\prime}}} \frac{a_{0}^{\varepsilon^{\prime}} \circ \sigma_{0}}{h_{0}^{\varepsilon^{\prime}} \circ \sigma_{0}} G_{0}\left(h_{0}^{\varepsilon^{\prime}} \circ \sigma_{0} H_{0}^{o}\left(\nabla u^{\varepsilon^{\prime}}\right)\right) \mathrm{d} x \geqslant \int_{\bar{\Gamma}_{0}} a_{0} H_{0}^{o}(n) G_{0}(|u|) \mathrm{d} s .
$$

Proof. - We recall that the injection mapping: $W^{1, p}(\Omega) \hookrightarrow L^{p}(\Omega)$ is compact and that the trace mappings $v \in W^{1, p}(\Omega) \rightarrow v_{\mid \partial \Gamma} \in L^{p}(\partial \Gamma)$ or $v_{\mid \partial \Gamma_{0}} \in L^{p}\left(\partial \Gamma_{0}\right)$ also are compact. Hence all the convergences follow from Lemma 1 and from compactness and we know that $u \in K, u_{\mid \Gamma} \in L^{q}(\Gamma)$ and $u_{\mid \Gamma_{0}} \in L^{q_{0}}\left(\Gamma_{0}\right)$. It just remains to prove that $u_{\mid \underline{\Gamma}}=k$ if $\underline{\Gamma} \neq \emptyset, u_{\mid \underline{\Gamma}_{0}}=0$ if $\underline{\Gamma}_{0} \neq \emptyset$, and to prove (4.8), (4.9).

$\star$ We begin by recalling the following properties of the distance function $t$. (These properties are already mentionned in [5], where a proof is given.)

$$
\begin{gathered}
\nabla t(x) . n_{H}(\sigma(x))=1, \\
H^{o}(\nabla t(x))=1, \\
\frac{1}{|\nabla t(x)|}=H^{o}(n(x)),
\end{gathered}
$$

where $n(x)$ is the normal at $x$ to the hypersurface $t=t(x)$. Of course one has similar versions of (4.10) to (4.12) with subscripts zero.

$\star$ For any $v \in \mathcal{C}^{1}\left(\overline{\Sigma^{\varepsilon}}\right), v_{\mid \Gamma^{\varepsilon}}=l$ (constant), we have, using the diffeomorphism of $\Sigma^{\prime}$ on $\partial \Omega \times\left(0, t^{\prime}\right)$, refining an argument already used above and denoting $s_{t}=s+t n_{H}(s)$,

$$
\int_{\Sigma^{\varepsilon}} \frac{a^{\varepsilon} \circ \sigma}{h^{\varepsilon} \circ \sigma} G\left(h^{\varepsilon} \circ \sigma H^{o}(\nabla v)\right) \mathrm{d} x=\int_{\Gamma} \int_{0}^{h^{\varepsilon}(s)} \frac{a^{\varepsilon}(s)}{h^{\varepsilon}(s)} G\left(h^{\varepsilon}(s) H^{o}\left(\nabla v\left(s_{t}\right)\right) \psi(s, t) \mathrm{d} t \mathrm{~d} s\right.
$$

and

$$
\psi(s, t)=\psi(s, 0)+\frac{\partial \psi}{\partial t}(s, \theta(s, t)) t=\frac{1}{|\nabla t|}+\Phi(s, t) t=H^{o}(n)+\Phi(s, t) t
$$

by (4.12), where $n$ is the normal to $\Gamma$ and where $\Phi$ is bounded $(|\Phi| \leqslant M)$. Hence

$$
\begin{aligned}
\int_{\Sigma^{\varepsilon}} \frac{a^{\varepsilon} \circ \sigma}{h^{\varepsilon} \circ \sigma} G\left(h^{\varepsilon} \circ \sigma H^{o}(\nabla v)\right) \mathrm{d} x \\
\quad=\int_{\Gamma} \int_{0}^{h^{\varepsilon}(s)} \frac{a^{\varepsilon}(s)}{h^{\varepsilon}(s)} G\left(h^{\varepsilon}(s) H^{o}\left(\nabla v\left(s_{t}\right)\right)\right) H^{o}(n) \mathrm{d} t \mathrm{~d} s+A^{\varepsilon}
\end{aligned}
$$

with

$$
\begin{aligned}
\left|A^{\varepsilon}\right| & \leqslant \int_{\Gamma} \int_{0}^{h^{\varepsilon}(s)} \frac{a^{\varepsilon}(s)}{h^{\varepsilon}(s)} G\left(h^{\varepsilon}(s) H^{o}\left(\nabla v\left(s_{t}\right)\right)\right)|\Phi(s, t)| t \mathrm{~d} t \mathrm{~d} s \\
& \leqslant \varepsilon M \int_{\Gamma}^{h^{\varepsilon}(s)} \int_{0}^{\varepsilon} \frac{a^{\varepsilon}(s)}{h^{\varepsilon}(s)} G\left(h^{\varepsilon}(s) H^{o}\left(\nabla v\left(s_{t}\right)\right)\right) \mathrm{d} t \mathrm{~d} s
\end{aligned}
$$


(as $|\Phi| \leqslant M$ and $0 \leqslant t \leqslant h^{\varepsilon}(s) \leqslant \varepsilon$ )

$$
\leqslant C \varepsilon M \int_{\Sigma^{\varepsilon}} \frac{a^{\varepsilon} \circ \sigma}{h^{\varepsilon} \circ \sigma} G\left(h^{\varepsilon} \circ \sigma H^{o}(\nabla v)\right) \mathrm{d} x .
$$

We get from (4.13)

$$
\begin{aligned}
& \int_{\Gamma} \int_{0}^{h^{\varepsilon}(s)} \frac{a^{\varepsilon}(s)}{h^{\varepsilon}(s)} G\left(h^{\varepsilon}(s) H^{o}\left(\nabla v\left(s_{t}\right)\right)\right) H^{o}(n) \mathrm{d} t \mathrm{~d} s \\
& \quad \leqslant(1+C \varepsilon M) \int_{\Sigma^{\varepsilon}} \frac{a^{\varepsilon} \circ \sigma}{h^{\varepsilon} \circ \sigma} G\left(h^{\varepsilon} \circ \sigma H^{o}(\nabla v)\right) \mathrm{d} x .
\end{aligned}
$$

As in [5]

$$
|v(s)-l| \leqslant \int_{0}^{h^{\varepsilon}(s)} H^{o}\left(\nabla v\left(s_{t}\right)\right) \mathrm{d} t
$$

and as $G$ is monotone nondecreasing

$$
\begin{aligned}
G(|v(s)-l|) & \leqslant G\left(\int_{0}^{h^{\varepsilon}(s)} H^{o}\left(\nabla v\left(s_{t}\right)\right) \mathrm{d} t\right)=G\left(\frac{1}{h^{\varepsilon}(s)} \int_{0}^{h^{\varepsilon}(s)} h^{\varepsilon}(s) H^{o}\left(\nabla v\left(s_{t}\right)\right) \mathrm{d} t\right) \\
& \leqslant \frac{1}{h^{\varepsilon}(s)} \int_{0}^{h^{\varepsilon}(s)} G\left(h^{\varepsilon}(s) H^{o}\left(\nabla v\left(s_{t}\right)\right)\right) \mathrm{d} t
\end{aligned}
$$

(by Jensen inequality). It follows, by means of (4.14),

$$
\begin{aligned}
& \int_{\Gamma} a^{\varepsilon}(s) G(|v(s)-l|) H^{o}(n) \mathrm{d} s \\
& \quad=\int_{\Gamma} \frac{a^{\varepsilon}(s)}{h^{\varepsilon}(s)} h^{\varepsilon}(s) G(|v(s)-l|) H^{o}(n) \mathrm{d} s \\
& \quad \leqslant \int_{\Gamma} \frac{a^{\varepsilon}(s)}{h^{\varepsilon}(s)}\left(\int_{0}^{h^{\varepsilon}(s)} G\left(h^{\varepsilon}(s) H^{o}\left(\nabla v\left(s_{t}\right)\right)\right) \mathrm{d} t\right) H^{o}(n) \mathrm{d} s \\
& \quad \leqslant(1+C \varepsilon M) \int_{\Sigma^{\varepsilon}} \frac{a^{\varepsilon} \circ \sigma}{h^{\varepsilon} \circ \sigma} G\left(h^{\varepsilon} \circ \sigma H^{o}(\nabla v)\right) \mathrm{d} x .
\end{aligned}
$$

This is also true by density for any $v \in W^{1, q}\left(\Sigma^{\varepsilon}\right)$ such that $v_{\mid \Gamma^{\varepsilon}}=l$ and in particular for $u^{\varepsilon}$, with $u_{\mid \Gamma^{\varepsilon}}^{\varepsilon}$ denoted by $k^{\varepsilon}$ :

$$
\int_{\Gamma} a^{\varepsilon}(s) G\left(\left|u^{\varepsilon}-k^{\varepsilon}\right|\right) H^{o}(n) \mathrm{d} s
$$




$$
\begin{aligned}
& \leqslant(1+C \varepsilon M) \int_{\Sigma^{\varepsilon}} \frac{a^{\varepsilon} \circ \sigma}{h^{\varepsilon} \circ \sigma} G\left(h^{\varepsilon} \circ \sigma H^{o}\left(\nabla u^{\varepsilon}\right)\right) \mathrm{d} x \\
& \leqslant C(1+C \varepsilon M)(\text { by Lemma } 1) \leqslant C,
\end{aligned}
$$

which gives from (2.5), (2.8), (2.10)

$$
\begin{aligned}
\int_{\underline{\Gamma}} \mid & u^{\varepsilon}(s)-k^{\varepsilon} \mid \mathrm{d} s \\
& =\int_{\Gamma}\left(a^{\varepsilon}(s)\right)^{-1 / q}\left(a^{\varepsilon}(s)\right)^{1 / q}\left|u^{\varepsilon}(s)-k^{\varepsilon}\right| \mathrm{d} s \\
& \leqslant\left(\int_{\Gamma}\left(a^{\varepsilon}(s)\right)^{-q^{\prime} / q} \mathrm{~d} s\right)^{1 / q^{\prime}}\left(\int_{\underline{\Gamma}} a^{\varepsilon}(s)\left|u^{\varepsilon}(s)-k^{\varepsilon}\right|^{q} \mathrm{~d} s\right)^{1 / q} \\
\leqslant & \left(\int_{\Gamma}\left[\left(a^{\varepsilon}(s)\right)^{-1}\right]^{q^{\prime}-1} \mathrm{~d} s\right)^{1 / q^{\prime}}\left(\mu_{1}\right)^{-1 / q} \delta_{2}^{1 / q}\left(\int_{\underline{\Gamma}} a^{\varepsilon}(s) G\left(\left|u^{\varepsilon}(s)-k^{\varepsilon}\right|\right) H^{o}(n) \mathrm{d} s\right)^{1 / q} \\
\leqslant & C\left(\int_{\Gamma}\left[\left(a^{\varepsilon}(s)\right)^{-1}\right]^{q^{\prime}-1} \mathrm{~d} s\right)^{1 / q^{\prime}} \rightarrow 0 .
\end{aligned}
$$

As $u^{\varepsilon^{\prime}} \rightarrow u_{\mid \underline{\Gamma}}$ in (strong) $L^{p}(\underline{\Gamma})$ and as $k^{\varepsilon^{\prime}} \rightarrow k$, we get $u_{\mid \underline{\Gamma}}=k$. By the same way one can prove that $u_{\mid \underline{\Sigma}_{0}}=0$.

$\star$ Now we prove (4.8). This is trivial if $\bar{\Gamma}$ is empty and is easy to prove from (4.15) if $q \leqslant p$ since then $u^{\varepsilon^{\prime}}{ }_{\mid \Gamma} \rightarrow u_{\mid \Gamma}$ in (strong-) $L^{q}(\Gamma)$ and $k^{\varepsilon^{\prime}} \rightarrow k$ imply that $G\left(\left|u^{\varepsilon^{\prime}}{ }_{\mid \Gamma}-k^{\varepsilon^{\prime}}\right|\right) \rightarrow G\left(\left|u_{\mid \Gamma}-k\right|\right)$ in (strong-) $L^{1}(\Gamma)$, so that as $a^{\varepsilon} \rightarrow a$ in weak $\star-L^{\infty}(\bar{\Gamma})$

$$
\begin{aligned}
\int_{\bar{\Gamma}} a G(|u-k|) H^{o}(n) \mathrm{d} s & =\lim \int_{\bar{\Gamma}} a^{\varepsilon^{\prime}} G\left(\left|u^{\varepsilon^{\prime}}-k^{\varepsilon^{\prime}}\right|\right) H^{o}(n) \mathrm{d} s \\
& \leqslant \liminf \int_{\Sigma^{\varepsilon^{\prime}}} \frac{a^{\varepsilon^{\prime}} \circ \sigma}{h^{\varepsilon^{\prime}} \circ \sigma} G\left(h^{\varepsilon^{\prime}} \circ \sigma H^{o}\left(\nabla u^{\varepsilon^{\prime}}\right)\right) \mathrm{d} x \quad \text { by (4.15). }
\end{aligned}
$$

If $\bar{\Gamma}$ is not empty and $q>p$, the proof (also valid otherwise) goes as follows. By convexity

$$
\begin{aligned}
G\left(\left|u^{\varepsilon}-k^{\varepsilon}\right|\right) & \geqslant G(|u-k|)+D\left(\left|u^{\varepsilon}-k^{\varepsilon}\right|-|u-k|\right) \\
& \geqslant G(|u-k|)-D\left|u^{\varepsilon}-k^{\varepsilon}-u+k\right|,
\end{aligned}
$$

for any $D \in \partial G(|u-k|)$, the subdifferential of $G$ at the point $|u-k|$. We notice that (2.8) and the monotonicity of $G$ imply

$$
\exists \mu>0, \quad \forall \xi \in \mathbb{R}^{+}, \quad \forall d \in \partial G(\xi), \quad 0 \leqslant d \leqslant \mu \xi^{q-1}
$$

and hence $D \in L^{q^{\prime}}(\Gamma)$, 


$$
\begin{aligned}
\int_{\Gamma} a^{\varepsilon^{\prime}} G\left(\left|u^{\varepsilon^{\prime}}-k^{\varepsilon^{\prime}}\right|\right) H^{o}(n) \mathrm{d} s \\
\geqslant \int_{\bar{\Gamma}} a^{\varepsilon^{\prime}} G\left(\left|u^{\varepsilon^{\prime}}-k^{\varepsilon^{\prime}}\right|\right) H^{o}(n) \mathrm{d} s \\
\geqslant \int_{\frac{\Gamma}{\Gamma}} a^{\varepsilon^{\prime}} G(|u-k|) H^{o}(n) \mathrm{d} s-\int_{\bar{\Gamma}} D a^{\varepsilon^{\prime}}\left|u^{\varepsilon^{\prime}}-k^{\varepsilon^{\prime}}-u+k\right| H^{o}(n) \mathrm{d} s .
\end{aligned}
$$

Now

$$
\int_{\bar{\Gamma}} a^{\varepsilon^{\prime}} G(|u-k|) H^{o}(n) \mathrm{d} s \rightarrow \int_{\bar{\Gamma}} a G(|u-k|) H^{o}(n) \mathrm{d} s .
$$

Moreover

$$
\int_{\bar{\Gamma}} D a^{\varepsilon^{\prime}}\left|u^{\varepsilon^{\prime}}-k^{\varepsilon^{\prime}}-u+k\right| H^{o}(n) \mathrm{d} s \rightarrow 0
$$

since $D H^{o}(n)$ belongs to $L^{q^{\prime}}(\bar{\Gamma})$ and $a^{\varepsilon^{\prime}}\left|u^{\varepsilon^{\prime}}-k^{\varepsilon^{\prime}}-u+k\right| \rightarrow 0$ in weak- $L^{q}(\bar{\Gamma})$, because $a^{\varepsilon^{\prime}}\left|u^{\varepsilon^{\prime}}-k^{\varepsilon^{\prime}}-u+k\right|$ is bounded in $L^{q}(\bar{\Gamma})$ and $a^{\varepsilon^{\prime}}\left|u^{\varepsilon^{\prime}}-k^{\varepsilon^{\prime}}-u+k\right| \rightarrow 0$ in (strong) $L^{p}(\bar{\Gamma})$. Therefore by $(4.15)$

$$
\begin{aligned}
\liminf \int_{\Sigma^{\varepsilon^{\prime}}} \frac{a^{\varepsilon^{\prime}} \circ \sigma}{h^{\varepsilon^{\prime}} \circ \sigma} G\left(h^{\varepsilon^{\prime}} \circ \sigma H^{o}\left(\nabla u^{\varepsilon^{\prime}}\right)\right) \mathrm{d} x & \geqslant \liminf \int_{\Gamma} a^{\varepsilon^{\prime}} G\left(\left|u^{\varepsilon^{\prime}}-k^{\varepsilon^{\prime}}\right|\right) H^{o}(n) \mathrm{d} s \\
& \geqslant \int_{\bar{\Gamma}} a G(|u-k|) H^{o}(n) \mathrm{d} \sigma .
\end{aligned}
$$

Inequality (4.9) is proved similarly (see also [5]).

\section{Proof of the convergence of $\left(\mathcal{P}^{\varepsilon}\right)$ to $(\mathcal{P})$}

We first prove it from Lemma 3, whose proof is postponed.

LEMMA 3. - Let $v \in \mathcal{C}^{1}(\bar{\Omega}) \cap K, v_{\mid \Gamma_{0}}=0$ and let $l \in \mathbb{R}$. If $\underline{\Gamma} \neq \emptyset$, we assume that $v_{\mid \underline{\Gamma}}=l$, otherwise there is no additional restriction on $v$ and $l$. For any such $v$ and $l$, there exists a sequence of elements $v^{\varepsilon} \in V^{\varepsilon}$ such that $v_{\mid \Omega}^{\varepsilon} \in K$ and

$$
\begin{gathered}
v_{\mid \Omega}^{\varepsilon}=v, \quad v_{\mid \Gamma^{\varepsilon}}^{\varepsilon}=l, \quad\left\|v^{\varepsilon}-l\right\|_{L^{q}\left(\Sigma^{\varepsilon}\right)} \rightarrow 0, \quad v_{\mid \Gamma_{0}^{\varepsilon}}^{\varepsilon}=0, \quad\left\|v^{\varepsilon}\right\|_{L^{q_{0}\left(\Sigma_{0}^{\varepsilon}\right)}} \rightarrow 0, \\
\limsup \int_{\Sigma^{\varepsilon}} \frac{a^{\varepsilon} \circ \sigma}{h^{\varepsilon} \circ \sigma} G\left(h^{\varepsilon} \circ \sigma H^{o}\left(\nabla v^{\varepsilon}\right)\right) \mathrm{d} x \leqslant \int_{\bar{\Gamma}} a G(|v-l|) H^{o}(n) \mathrm{d} s, \\
\limsup \int_{\Sigma_{0}^{\varepsilon}} \frac{a_{0}^{\varepsilon} \circ \sigma_{0}}{h_{0}^{\varepsilon} \circ \sigma_{0}} G_{0}\left(h_{0}^{\varepsilon} \circ \sigma_{0} H_{0}^{o}\left(\nabla v^{\varepsilon}\right)\right) \mathrm{d} x \leqslant \int_{\bar{\Gamma}_{0}} a_{0} G_{0}(|v|) H^{o}(n) \mathrm{d} s .
\end{gathered}
$$


Proof of Theorem 1 assuming Lemma 3. - Let $u^{\varepsilon}$ be the solution of $\left(\mathcal{P}^{\varepsilon}\right)$. From Lemma 1,

$$
\left.\int_{\Sigma^{\varepsilon}}\left|u^{\varepsilon}-u^{\varepsilon}\right| \Gamma^{\varepsilon}\right|^{q} \mathrm{~d} x \quad \text { and } \quad \int_{\Sigma_{0}^{\varepsilon}}\left|u^{\varepsilon}\right|^{q_{0}} \mathrm{~d} x \rightarrow 0,
$$

so that (2) is proved. Let $(u, k) \in W$ and $\varepsilon^{\prime}$ be as in Lemma 2. As the solution of $(\mathcal{P})$ is unique it just remains to prove that $(u, k)$ solves $(\mathcal{P})$ and that $J^{\varepsilon^{\prime}}\left(u^{\varepsilon^{\prime}}\right) \rightarrow J(u, k)$. (Then a classical argument gives $\varepsilon^{\prime} \equiv \varepsilon$ : one has the convergences for the whole sequence $\varepsilon$.) Let $v \in \mathcal{C}^{1}(\bar{\Omega}) \cap K, v_{\mid \underline{\Sigma}_{0}}=0$ and let $l \in \mathbb{R}$. If $\underline{\Gamma} \neq \emptyset$, assume that $v_{\mid \underline{\Gamma}}=l$. Finally let $v^{\varepsilon}$ be as in Lemma 3.

(A) $\liminf J^{\varepsilon^{\prime}}\left(u^{\varepsilon^{\prime}}\right) \leqslant \limsup J^{\varepsilon^{\prime}}\left(u^{\varepsilon^{\prime}}\right) \leqslant \limsup J^{\varepsilon^{\prime}}\left(v^{\varepsilon^{\prime}}\right)$

$$
\begin{aligned}
& =\limsup \left\{F(v)+\int_{\Sigma^{\varepsilon^{\prime}}} \frac{a^{\varepsilon^{\prime}} \circ \sigma}{h^{\varepsilon^{\prime}} \circ \sigma} G\left(h^{\varepsilon^{\prime}} \circ \sigma H^{o}\left(\nabla v^{\varepsilon^{\prime}}\right)\right) \mathrm{d} x\right. \\
& +\int_{\Sigma_{0}^{\varepsilon^{\prime}}} \frac{a_{0}^{\varepsilon^{\prime}} \circ \sigma_{0}}{h_{0}^{\varepsilon^{\prime}} \circ \sigma_{0}} G_{0}\left(h_{0}^{\varepsilon^{\prime}} \circ \sigma_{0} H_{0}^{o}\left(\nabla v^{\varepsilon^{\prime}}\right)\right) \mathrm{d} x \\
& \left.-\int_{\Omega} f^{\varepsilon^{\prime}} v^{\varepsilon^{\prime}} \mathrm{d} x-\int_{\Sigma^{\varepsilon^{\prime}}} g^{\varepsilon^{\prime}} v^{\varepsilon^{\prime}} \mathrm{d} x-\int_{\Sigma_{0}^{\varepsilon^{\prime}}} g_{0}^{\varepsilon^{\prime}} v^{\varepsilon^{\prime}} \mathrm{d} x-I^{\varepsilon^{\prime}} l\right\} .
\end{aligned}
$$

But $\int_{\Omega} f^{\varepsilon^{\prime}} v \mathrm{~d} x \rightarrow \int_{\Omega} f v \mathrm{~d} x$, since $f^{\varepsilon^{\prime}} \rightarrow f$ in weak- $L^{p^{\prime}}(\Omega), v \in L^{p}(\Omega)$, and $I^{\varepsilon^{\prime}} \rightarrow$ $I$ in $\mathbb{R}$. Moreover

$$
\begin{aligned}
\left|\int_{\Sigma^{\varepsilon^{\prime}}} g^{\varepsilon^{\prime}} v^{\varepsilon^{\prime}} \mathrm{d} x\right| & =\left|\int_{\Sigma^{\varepsilon^{\prime}}} g^{\varepsilon^{\prime}}\left(v^{\varepsilon^{\prime}}-l+l\right) \mathrm{d} x\right| \\
& \leqslant\left\|g^{\varepsilon^{\prime}}\right\|_{L^{q^{\prime}}\left(\Sigma^{\varepsilon^{\prime}}\right)}\left(\left\|v^{\varepsilon^{\prime}}-l\right\|_{L^{q}\left(\Sigma^{\varepsilon^{\prime}}\right)}+|l|\left|\Sigma^{\varepsilon^{\prime}}\right|^{1 / q}\right) \\
& \leqslant C\left(\left\|v^{\varepsilon^{\prime}}-l\right\|_{L^{q}\left(\Sigma^{\varepsilon^{\prime}}\right)}+|l|\left|\Sigma^{\varepsilon^{\prime}}\right|^{1 / q}\right)
\end{aligned}
$$

(by (2.14)), which tends to zero thanks to (5.1). Similarly,

$$
\int_{\Sigma_{0}^{\varepsilon^{\prime}}} g_{0}^{\varepsilon^{\prime}} v^{\varepsilon^{\prime}} \mathrm{d} x \rightarrow 0
$$

so that by Lemma 3,

$$
\begin{aligned}
\liminf J^{\varepsilon^{\prime}}\left(u^{\varepsilon^{\prime}}\right) \leqslant & \limsup J^{\varepsilon^{\prime}}\left(u^{\varepsilon^{\varepsilon^{\prime}}}\right) \\
\leqslant & F(v)-\int_{\Omega} f v \mathrm{~d} x-I l+\lim \sup \int_{\Sigma^{\varepsilon^{\prime}}} \frac{a^{\varepsilon^{\prime}} \circ \sigma}{h^{\varepsilon^{\prime}} \circ \sigma} G\left(h^{\varepsilon^{\prime}} \circ \sigma H^{o}\left(\nabla v^{\varepsilon^{\prime}}\right)\right) \mathrm{d} x \\
& +\limsup \int_{\Sigma_{0}^{\varepsilon^{\prime}}} \frac{a_{0}^{\varepsilon^{\prime}} \circ \sigma_{0}}{h_{0}^{\varepsilon^{\prime}} \circ \sigma_{0}} G_{0}\left(h_{0}^{\varepsilon^{\prime}} \circ \sigma_{0} H_{0}^{o}\left(\nabla v^{\varepsilon^{\prime}}\right)\right) \mathrm{d} x
\end{aligned}
$$


M. BOUTKRIDA ET AL. / Ann. I. H. Poincaré - AN 19 (2002) 13-40

$$
\begin{aligned}
& \leqslant F(v)-\int_{\Omega} f v \mathrm{~d} x-I l \\
& \quad+\int_{\bar{\Gamma}} a G(|v-l|) H^{o}(n) \mathrm{d} s+\int_{\Gamma_{0}} a_{0} G_{0}(|v|) H_{0}^{o}(n) \mathrm{d} s \\
& =J(v, l) .
\end{aligned}
$$

$$
\text { (B) } \begin{aligned}
J^{\varepsilon^{\prime}}\left(u^{\varepsilon^{\prime}}\right)= & F\left(u^{\varepsilon^{\prime}}{ }_{\mid \Omega}\right)+\int_{\Sigma^{\varepsilon^{\prime}}} \frac{a^{\varepsilon^{\prime}} \circ \sigma}{h^{\varepsilon^{\prime}} \circ \sigma} G\left(h^{\varepsilon^{\prime}} \circ \sigma H^{o}\left(\nabla u^{\varepsilon^{\prime}}\right)\right) \mathrm{d} x \\
& +\int_{\Sigma_{0}^{\varepsilon^{\prime}}} \frac{a_{0}^{\varepsilon^{\prime}} \circ \sigma_{0}}{h_{0}^{\varepsilon^{\prime}} \circ \sigma_{0}} G_{0}\left(h_{0}^{\varepsilon^{\prime}} \circ \sigma_{0} H_{0}^{o}\left(\nabla u^{\varepsilon^{\prime}}\right)\right) \mathrm{d} x \\
& -\int_{\Omega} f^{\varepsilon^{\prime}} u^{\varepsilon^{\prime}} \mathrm{d} x-\int_{\Sigma^{\varepsilon^{\prime}}} g^{\varepsilon^{\prime}} u^{\varepsilon^{\prime}} \mathrm{d} x-\int_{\Sigma_{0}^{\varepsilon^{\prime}}} g_{0}^{\varepsilon^{\prime}} u^{\varepsilon^{\prime}} \mathrm{d} x-I^{\varepsilon^{\prime}} u_{\mid \Gamma^{\varepsilon^{\prime}}}^{\varepsilon^{\prime}}
\end{aligned}
$$

As above

$$
\left|\int_{\Sigma^{\varepsilon^{\prime}}} g^{\varepsilon^{\prime}} u^{\varepsilon^{\prime}} \mathrm{d} x\right| \leqslant C\left(\left\|u^{\varepsilon^{\prime}}-u_{\mid \Gamma^{\varepsilon^{\varepsilon^{\prime}}}}^{\varepsilon^{\prime}}\right\|_{L^{q}\left(\Sigma^{\varepsilon^{\prime}}\right)}+\left|u_{\mid \Gamma^{\varepsilon^{\prime}}}^{\varepsilon^{\prime}}\right|\left|\Sigma^{\varepsilon^{\prime}}\right|^{1 / q}\right)
$$

tends to zero. Of course the same holds for the corresponding integral over $\Sigma_{0}^{\varepsilon^{\prime}}$. Then from Lemma 2 and (2.14) we get

$$
\begin{aligned}
\liminf J^{\varepsilon^{\prime}}\left(u^{\varepsilon^{\prime}}\right) \geqslant & \liminf F\left(u^{\varepsilon^{\prime}} \mid \Omega\right)+\liminf \int_{\Sigma^{\varepsilon^{\prime}}} \frac{a^{\varepsilon^{\prime}} \circ \sigma}{h^{\varepsilon^{\prime}} \circ \sigma} G\left(h^{\varepsilon^{\prime}} \circ \sigma H^{o}\left(\nabla u^{\varepsilon^{\prime}}\right)\right) \mathrm{d} x \\
& +\liminf \int_{\Sigma_{0}^{\varepsilon^{\prime}}} \frac{a_{0}^{\varepsilon^{\prime}} \circ \sigma_{0}}{h_{0}^{\varepsilon^{\prime}} \circ \sigma_{0}} G_{0}\left(h_{0}^{\varepsilon^{\prime}} \circ \sigma_{0} H_{0}^{o}\left(\nabla u^{\varepsilon^{\prime}}\right)\right) \mathrm{d} x-\int f u \mathrm{~d} x-I k \\
\geqslant & F(u)+\int_{\Omega} a G(|u-k|) H^{o}(n) \mathrm{d} s+\int_{\Gamma_{0}} a_{0} G_{0}(|u|) H_{0}^{o}(n) \mathrm{d} s \\
& -\int_{\Omega} f u \mathrm{~d} x-I k \\
= & J(u, k)
\end{aligned}
$$

(by using the lower semi-continuity of $F$, the weak- $W^{1, p}(\Omega)$ convergence of $u^{\varepsilon^{\prime}}{ }_{\mid \Omega}$ and (4.8)).

(C) From (A) and (B): Fo r any $l \in \mathbb{R}, v \in \mathcal{C}^{1}(\bar{\Omega}) \cap K$ such that $v_{\mid \underline{\Gamma}_{0}}=0$ and $v_{\mid \underline{\Gamma}}=l$

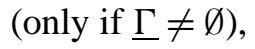

$$
J(u, k) \leqslant \liminf J^{\varepsilon^{\prime}}\left(u^{\varepsilon^{\prime}}\right) \leqslant \limsup J^{\varepsilon^{\prime}}\left(u^{\varepsilon^{\prime}}\right) \leqslant J(v, l) .
$$


By the density property (2.13) and by continuity, this is also true for any $(v, l) \in W, v \in$ $K$, that is $(u, k)$ solves $(\mathcal{P})$ and $J^{\varepsilon^{\prime}}\left(u^{\varepsilon^{\prime}}\right)$ tends to $J(u, k)$. This completes the proof of Theorem 1, except that we have to prove Lemma 3.

Proof of Lemma 3. - Let $v \in \mathcal{C}^{1}(\bar{\Omega}) \cap K, v_{\mid \underline{\Gamma}_{0}}=0$ and let $l \in \mathbb{R}$. If $\underline{\Gamma} \neq \emptyset$, we assume that $v_{\mid \underline{\Gamma}}=l$. Let $v^{\varepsilon}$ be the continuous function defined in $\bar{\Omega}^{\varepsilon}$ by

$$
v^{\varepsilon}(x)= \begin{cases}v(x) & \text { if } x \in \Omega, \\ w(x) \varphi^{\varepsilon}(x)+l\left(1-\varphi^{\varepsilon}(x)\right) & \text { if } x \in \bar{\Sigma}^{\varepsilon}, \\ w_{0}(x) \varphi_{0}^{\varepsilon}(x) & \text { if } x \in \bar{\Sigma}_{0}^{\varepsilon},\end{cases}
$$

with $w(x)=(v \circ \sigma)(x), w_{0}(x)=\left(v \circ \sigma_{0}\right)(x), \varphi^{\varepsilon}(x)=1-\frac{t(x)}{\left(h^{\varepsilon} \circ \sigma\right)(x)}, \varphi_{0}^{\varepsilon}(x)=1-\frac{t_{0}(x)}{\left(h_{0}^{\varepsilon} \circ \sigma_{0}\right)(x)}$, so that $v^{\varepsilon}=v$ in $\bar{\Omega}, v^{\varepsilon}=l$ on $\Gamma^{\varepsilon}, v^{\varepsilon}=0$ on $\Gamma_{0}^{\varepsilon}$. Clearly $v^{\varepsilon} \in V^{\varepsilon}$. Moreover

$$
\left\|v^{\varepsilon}-l\right\|_{L^{q}\left(\Sigma^{\varepsilon}\right)}^{q}=\left\|(w-l) \varphi^{\varepsilon}\right\|_{L^{q}\left(\Sigma^{\varepsilon}\right)}^{q} \leqslant \int_{\Sigma^{\varepsilon}}|w-l|^{q} \mathrm{~d} x \rightarrow 0,
$$

since $w-l$ is bounded and since $\left|\Sigma^{\varepsilon}\right|$ tends to zero. Similarly $\left\|v^{\varepsilon}\right\|_{L^{q_{0}}\left(\Sigma_{0}^{\varepsilon}\right)}$ tends to zero. It just remains to prove the inequalities (5.2), (5.3) in Lemma 3. In the following we consider only (5.2), since the proof of (5.3) is very similar and was published in [5]. One writes for simplicity

$$
K^{\varepsilon}(x, \xi)=\frac{a^{\varepsilon} \circ \sigma}{h^{\varepsilon} \circ \sigma}(x) G\left(\left(h^{\varepsilon} \circ \sigma\right)(x) H^{o}(\xi)\right),
$$

so that, denoting $\Sigma_{r}^{\varepsilon}=\left\{s+t n_{H}(s), s \in \bar{\Gamma}, 0<t<h^{\varepsilon}(s)\right\}$ the reduced part of $\Sigma^{\varepsilon}$ with $H$-projection in $\bar{\Gamma}$,

$$
\int_{\Sigma^{\varepsilon}} \frac{a^{\varepsilon} \circ \sigma}{h^{\varepsilon} \circ \sigma} G\left(h^{\varepsilon} \circ \sigma H^{o}\left(\nabla v^{\varepsilon}\right)\right) \mathrm{d} x=\int_{\Sigma_{r}^{\varepsilon}} K^{\varepsilon}\left(x, \nabla v^{\varepsilon}\right) \mathrm{d} x,
$$

where $K^{\varepsilon}$ is convex in $\xi$. Using a classical convexity argument valid for any $\theta \in(0,1)$,

$$
\begin{aligned}
\int_{\Sigma_{r}^{\varepsilon}} K^{\varepsilon}\left(x, \nabla v^{\varepsilon}\right) \mathrm{d} x & =\int_{\Sigma_{r}^{\varepsilon}} K^{\varepsilon}\left(x, \theta \varphi^{\varepsilon} \frac{\nabla w}{\theta}+(1-\theta)(w-l) \frac{\nabla \varphi^{\varepsilon}}{1-\theta}\right) \mathrm{d} x \\
& \leqslant \theta \int_{\Sigma_{r}^{\varepsilon}} K^{\varepsilon}\left(x, \varphi^{\varepsilon} \frac{\nabla w}{\theta}\right) \mathrm{d} x+(1-\theta) \int_{\Sigma_{2}^{\varepsilon}} K^{\varepsilon}\left(x,(w-l) \frac{\nabla \varphi^{\varepsilon}}{1-\theta}\right) \mathrm{d} x .
\end{aligned}
$$

By definition of $K^{\varepsilon}$ and by (2.5), (2.6), (2.8), (2.11),

$$
\begin{aligned}
& \theta \int_{\Sigma_{r}^{\varepsilon}} K^{\varepsilon}\left(x, \varphi^{\varepsilon} \frac{\nabla w}{\theta}\right) \mathrm{d} x=\theta \int_{\Sigma_{r}^{\varepsilon}} \frac{a^{\varepsilon} \circ \sigma}{h^{\varepsilon} \circ \sigma} G\left(h^{\varepsilon} \circ \sigma \frac{\varphi^{\varepsilon} H^{o}(\nabla w)}{\theta}\right) \mathrm{d} x \\
& \quad \leqslant \frac{\mu_{2}}{\delta_{1}^{q} \theta^{q-1}} \int_{\Sigma_{r}^{\varepsilon}}\left(a^{\varepsilon} \circ \sigma\right)\left(h^{\varepsilon} \circ \sigma\right)^{q-1}\left(\varphi^{\varepsilon}\right)^{q}|\nabla w|^{q} \mathrm{~d} x \leqslant \frac{C \mu_{2}}{\delta_{1}^{q} \theta^{q-1}} \varepsilon^{q-1} \int_{\Sigma_{r}^{\varepsilon}}|\nabla w|^{q} \mathrm{~d} x \rightarrow 0,
\end{aligned}
$$


since $\nabla w$ is bounded, so that

$$
\limsup \int_{\Sigma_{r}^{\varepsilon}} K^{\varepsilon}\left(x, \nabla v^{\varepsilon}\right) \mathrm{d} x \leqslant(1-\theta) \lim \sup \int_{\Sigma_{r}^{\varepsilon}} K^{\varepsilon}\left(x, \frac{(w-l) \nabla \varphi^{\varepsilon}}{1-\theta}\right) \mathrm{d} x
$$

for any $\theta \in(0,1)$ and letting $\theta$ tend to zero

$$
\limsup \int_{\Sigma_{r}^{\varepsilon}} K^{\varepsilon}\left(x, \nabla v^{\varepsilon}\right) \mathrm{d} x \leqslant \lim \sup \int_{\Sigma_{r}^{\varepsilon}} K^{\varepsilon}\left(x,(w-l) \nabla \varphi^{\varepsilon}\right) \mathrm{d} x .
$$

Now on $\Sigma_{r}^{\varepsilon}$, we have by the classical properties of the norm $H^{o}$ and (4.11)

$$
\begin{aligned}
H^{o}\left(\nabla \varphi^{\varepsilon}\right)=H^{o}\left(-\frac{\nabla t}{h^{\varepsilon} \circ \sigma}+\frac{t \nabla\left(h^{\varepsilon} \circ \sigma\right)}{\left(h^{\varepsilon} \circ \sigma\right)^{2}}\right) & \leqslant \frac{H^{o}(\nabla t)}{h^{\varepsilon} \circ \sigma}+\frac{t}{h^{\varepsilon} \circ \sigma} \frac{H^{o}\left(\nabla\left(h^{\varepsilon} \circ \sigma\right)\right)}{h^{\varepsilon} \circ \sigma} \\
& \leqslant \frac{1}{h^{\varepsilon} \circ \sigma}+\frac{H^{o}\left(\nabla\left(h^{\varepsilon} \circ \sigma\right)\right)}{h^{\varepsilon} \circ \sigma}
\end{aligned}
$$

which implies, by the same convexity argument and by monotonicity, that

$$
\begin{aligned}
& \int_{\Sigma_{r}^{\varepsilon}} K^{\varepsilon}\left(x,(w-l) \nabla \varphi^{\varepsilon}\right) \mathrm{d} x \leqslant \int_{\Sigma_{r}^{\varepsilon}} \frac{a^{\varepsilon} \circ \sigma}{h^{\varepsilon} \circ \sigma} G\left(|w-l|+|w-l| H^{o}\left(\nabla\left(h^{\varepsilon} \circ \sigma\right)\right)\right) \mathrm{d} x \\
& \quad \leqslant \theta \int_{\Sigma_{r}^{\varepsilon}} \frac{a^{\varepsilon} \circ \sigma}{h^{\varepsilon} \circ \sigma} G\left(\frac{|w-l|}{\theta}\right) \mathrm{d} x+(1-\theta) \int_{\Sigma_{r}^{\varepsilon}} \frac{a^{\varepsilon} \circ \sigma}{h^{\varepsilon} \circ \sigma} G\left(\frac{|w-l| H^{o}\left(\nabla\left(h^{\varepsilon} \circ \sigma\right)\right)}{1-\theta}\right) \mathrm{d} x .
\end{aligned}
$$

Using (2.5) and (2.8), the last integral is bounded by

$$
\begin{aligned}
\frac{\mu_{2}}{\delta_{1}^{q}(1-\theta)^{q}} \int_{\Sigma_{r}^{\varepsilon}} a^{\varepsilon} \circ \sigma \frac{\left|\nabla\left(h^{\varepsilon} \circ \sigma\right)\right|^{q}}{h^{\varepsilon} \circ \sigma}|w-l|^{q} \mathrm{~d} x & \leqslant \frac{C}{(1-\theta)^{q}} \int_{\Sigma_{2}^{\varepsilon}} a^{\varepsilon} \circ \sigma \frac{\left|\nabla\left(h^{\varepsilon} \circ \sigma\right)\right|^{q}}{h^{\varepsilon} \circ \sigma} \mathrm{d} x \\
& \leqslant C \int_{\bar{\Gamma}} a^{\varepsilon}\left|\nabla h^{\varepsilon}\right|^{q} \mathrm{~d} s
\end{aligned}
$$

and therefore by (2.11), (2.12) it tends to zero with $\varepsilon$ : one obtains by letting $\theta$ tend to one

$$
\lim \sup \int_{\Sigma_{r}^{\varepsilon}} K^{\varepsilon}\left(x,(w-l) \nabla \varphi^{\varepsilon}\right) \mathrm{d} x \leqslant \limsup \int_{\Sigma_{r}^{\varepsilon}} \frac{a^{\varepsilon} \circ \sigma}{h^{\varepsilon} \circ \sigma} G(|w-l|) \mathrm{d} x .
$$

Finally thanks to the usual diffeomorphism argument

$$
\begin{aligned}
\int_{\Sigma_{r}^{\varepsilon}} \frac{a^{\varepsilon} \circ \sigma}{h^{\varepsilon} \circ \sigma} G(|w-l|) \mathrm{d} x & =\int_{\bar{\Gamma}} \int_{0}^{h^{\varepsilon}(s)} \frac{a^{\varepsilon}(s)}{h^{\varepsilon}(s)} G(|v(s)-l|)\left(H^{o}(n(s))+\Phi(s, t) t\right) \mathrm{d} t \mathrm{~d} s \\
& =\int_{\bar{\Gamma}} a^{\varepsilon}(s) G(|v(s)-l|) b^{\varepsilon}(s) \mathrm{d} s,
\end{aligned}
$$


where

$$
b^{\varepsilon}(s)=\frac{1}{h^{\varepsilon}(s)} \int_{0}^{h^{\varepsilon}(s)}\left(H^{o}(n(s))+\Phi(s, t) t\right) \mathrm{d} t .
$$

As $b^{\varepsilon}$ is uniformly bounded and $b^{\varepsilon}(s) \rightarrow H^{o}(n(s))$ for a.e. $s$ of $\bar{\Gamma}$, we have $b^{\varepsilon} \rightarrow$ $H^{o}(n)$ in $L^{1}(\bar{\Gamma})$. Then, as $a^{\varepsilon} \rightarrow a$ in weak $\star-L^{\infty}(\bar{\Gamma})$ (see (2.11)), one obtains

$$
\int_{\Sigma_{r}^{\varepsilon}} \frac{a^{\varepsilon} \circ \sigma}{h^{\varepsilon} \circ \sigma} G(|w-l|) \mathrm{d} x \rightarrow \int_{\bar{\Gamma}} a G(|v-l|) H^{o}(n) \mathrm{d} s
$$

and by means of (5.5), (5.6) this implies

$$
\begin{aligned}
& \lim \sup \int_{\Sigma_{r}^{\varepsilon}} \frac{a^{\varepsilon} \circ \sigma}{h^{\varepsilon} \circ \sigma} G\left(h^{\varepsilon} \circ \sigma H^{o}\left(\nabla v^{\varepsilon}\right)\right) \mathrm{d} x \\
& \quad=\lim \sup \int_{\Sigma_{r}^{\varepsilon}} K^{\varepsilon}\left(x, \nabla v^{\varepsilon}\right) \mathrm{d} x \leqslant \int_{\bar{\Gamma}} a G(|v-l|) H^{o}(n) \mathrm{d} s
\end{aligned}
$$

and the proof is complete.

\section{Example}

For given $\Sigma^{\varepsilon}, \Sigma_{0}^{\varepsilon}$ and $\mu^{\varepsilon}$ (respectively $\mu_{0}^{\varepsilon}$ ) in $L^{\infty}(\Gamma)$ (respectively $L^{\infty}\left(\Gamma_{0}\right)$ ), let us consider the problem of minimizing on $\left\{v: \Omega^{\varepsilon} \rightarrow \mathbb{R}, v \in V^{\varepsilon}, v_{\mid \Omega} \in K\right\}$ the functional

$$
\begin{aligned}
J^{\varepsilon}(v)= & \frac{1}{p} \int_{\Omega}(A \nabla v, \nabla v)^{p / 2} \mathrm{~d} x+\int_{\Omega} \Psi(v) \mathrm{d} x+\frac{1}{q} \int_{\Sigma^{\varepsilon}} \mu^{\varepsilon} \circ \sigma(B \nabla v, \nabla v)^{q / 2} \mathrm{~d} x \\
& +\frac{1}{q_{0}} \int_{\Sigma_{0}^{\varepsilon}} \mu_{0}^{\varepsilon} \circ \sigma_{0}\left(B_{0} \nabla v, \nabla v\right)^{q_{0} / 2} \mathrm{~d} x-\int_{\Omega} f^{\varepsilon} v \mathrm{~d} x \\
& -\int_{\Sigma^{\varepsilon}} g^{\varepsilon} v \mathrm{~d} x-\int_{\Sigma_{0}^{\varepsilon}} g_{0}^{\varepsilon} v \mathrm{~d} x-I^{\varepsilon} v_{\mid \Gamma^{\varepsilon}},
\end{aligned}
$$

where $A, B$ and $B_{0}$ are given coercive symmetric matrices with constant coefficients, $\Psi: \mathbb{R} \rightarrow \mathbb{R}$ is of class $\mathcal{C}^{1}$, convex, nonnegative and satisfies the growth condition:

$$
\Psi(\theta) \leqslant C|\theta|^{p}+C^{\prime},
$$

for some positive constants $C$ and $C^{\prime}$. Denoting by $\phi$ the derivative of $\Psi$, it follows that $\phi$ satisfies a similar growth condition:

$$
|\phi(\theta)| \leqslant C|\theta|^{p-1}+C^{\prime} .
$$

Then it is classical that $v \rightarrow \Psi(v)$ is continuous from $L^{p}(\Omega)$ into $L^{1}(\Omega)$ and that $v \rightarrow \phi(v)$ is continuous from $L^{p}(\Omega)$ into $L^{p^{\prime}}(\Omega)$. 
For $\Sigma^{\varepsilon}$ (respectively $\Sigma_{0}^{\varepsilon}$ ) given, let us define $h^{\varepsilon}: \Gamma \rightarrow \mathbb{R}\left(\right.$ respectively $h_{0}^{\varepsilon}: \Gamma_{0} \rightarrow \mathbb{R}$ ) by

$$
\Sigma^{\varepsilon}=\left\{s+\theta B n(s), 0<\theta<h^{\varepsilon}(s)(B n(s), n(s))^{-1 / 2}\right\}
$$

(respectively $\left.\Sigma_{0}^{\varepsilon}=\left\{s+\theta B_{0} n(s), 0<\theta<h_{0}^{\varepsilon}(s)\left(B_{0} n(s), n(s)\right)^{-1 / 2}\right\}\right)$,

where $n(s)$ is the normal to $\Gamma$ (respectively $\Gamma_{0}$ ) at $s$, pointing outside $\Omega$. Now let us assume that

- $\forall \varepsilon, h^{\varepsilon} \in \mathcal{C}^{1}(\Gamma)$ and $\forall s \in \Gamma, 0<h^{\varepsilon}(s) \leqslant \varepsilon$ (respectively $h_{0}^{\varepsilon} \in \mathcal{C}^{1}\left(\Gamma_{0}\right), 0<h^{\varepsilon}(s) \leqslant$ $\varepsilon)$;

- $\exists \alpha>0$, a.e. $s \in \Gamma, \forall \varepsilon, \mu^{\varepsilon}(s) \geqslant \alpha\left(h^{\varepsilon}(s)\right)^{q-1}$ (respectively a.e. $s \in \Gamma, \mu_{0}^{\varepsilon}(s) \geqslant$ $\left.\alpha\left(h_{0}^{\varepsilon}(s)\right)^{q_{0}-1}\right)$

- there exists a partition $\underline{\Gamma}, \bar{\Gamma}$ of $\Gamma$ and a partition $\underline{\Gamma}_{0}, \bar{\Gamma}_{0}$ of $\Gamma_{0}$ such that

$$
\begin{gathered}
\int_{\underline{\Gamma}} h^{\varepsilon}\left(\mu^{\varepsilon}\right)^{1-q^{\prime}} \mathrm{d} s \rightarrow 0 \text { and } \int_{\bar{\Gamma}}\left|\nabla h^{\varepsilon}\right|^{q} \mathrm{~d} s \rightarrow 0 \\
\left(\text { respectively } \int_{\Gamma_{0}} h_{0}^{\varepsilon}\left(\mu_{0}^{\varepsilon}\right)^{1-q_{0}^{\prime}} \mathrm{d} s \rightarrow 0 \text { and } \int_{\bar{\Gamma}_{0}}\left|\nabla h_{0}^{\varepsilon}\right|^{q_{0}} \mathrm{~d} s \rightarrow 0\right), \\
\mu^{\varepsilon}\left(h^{\varepsilon}\right)^{1-q} \rightarrow a \text { in weak } \star-L^{\infty}(\bar{\Gamma})\left(\text { respectively } \mu_{0}^{\varepsilon}\left(h_{0}^{\varepsilon}\right)^{1-q_{0}} \rightarrow a_{0}\right. \\
\text { in weak } \left.\star-L^{\infty}\left(\bar{\Gamma}_{0}\right)\right) \text {, for some } a \text { in } L^{\infty}(\bar{\Gamma}) \text { and } a_{0} \text { in } L^{\infty}\left(\bar{\Gamma}_{0}\right) \text {. }
\end{gathered}
$$

(The data $K, I^{\varepsilon}, f^{\varepsilon}, g^{\varepsilon}, g_{0}^{\varepsilon}$ satisfy the general assumptions.)

In this case one can apply the abstract result given in Theorem 1 with

$$
\begin{gathered}
F(v)=\frac{1}{p} \int_{\Omega}(A \nabla v, \nabla v)^{p / 2} \mathrm{~d} x+\int_{\Omega} \Psi(v) \mathrm{d} x, \\
G(\eta)=\frac{1}{q} \eta^{q} \quad\left(\text { respectively } G_{0}(\eta)=\frac{1}{q_{0}} \eta^{q_{0}}\right), \\
H(\xi)=\left(B^{-1} \xi, \xi\right)^{1 / 2} \quad\left(\text { respectively } H_{0}(\xi)=\left(B_{0}^{-1} \xi, \xi\right)^{1 / 2}\right), \\
H^{o}\left(\xi^{o}\right)=\left(B \xi^{o}, \xi^{o}\right)^{1 / 2} \quad\left(\text { respectively } H_{0}^{o}\left(\xi^{o}\right)=\left(B_{0} \xi^{o}, \xi^{o}\right)^{1 / 2}\right),
\end{gathered}
$$

$n_{H}(s)=B n(s)(B n(s), n(s))^{-1 / 2} \quad\left(\right.$ respectively $\left.n_{H_{0}}(s)=B_{0} n(s)\left(B_{0} n(s), n(s)\right)^{-1 / 2}\right)$.

The application of Theorem 1 gives as limit problem

$$
\operatorname{Inf}\{J(v, l),(v, l) \in W v \in K\},
$$

where $W$ is as before and where 


$$
\begin{aligned}
J(v, l)= & \frac{1}{p} \int_{\Omega}(A \nabla v, \nabla v)^{p / 2} \mathrm{~d} x+\int_{\Omega} \Psi(v) \mathrm{d} x+\frac{1}{q} \int_{\bar{\Gamma}}(B n, n)^{1 / 2} a|v-l|^{q} \mathrm{~d} s \\
& +\frac{1}{q_{0}} \int_{\bar{\Gamma}_{0}}\left(B_{0} n, n\right)^{1 / 2} a_{0}|v|^{q_{0}} \mathrm{~d} s-\int_{\Omega} f v \mathrm{~d} x-I l .
\end{aligned}
$$

Let us interpret this result. The solution $u^{\varepsilon}$ of $\left(\mathcal{P}^{\varepsilon}\right)$ is characterized by the variational formulation:

$$
\begin{aligned}
& u^{\varepsilon} \in V^{\varepsilon}, u_{\mid \Omega}^{\varepsilon} \in K, \text { and for every } v \in V^{\varepsilon}, v_{\mid \Omega} \in K \\
& \int_{\Omega}\left(A \nabla u^{\varepsilon}, \nabla u^{\varepsilon}\right)^{\frac{p}{2}-1}\left(A \nabla u^{\varepsilon}, \nabla\left(v-u^{\varepsilon}\right)\right) \mathrm{d} x+\int_{\Omega} \phi\left(u^{\varepsilon}\right)\left(v-u^{\varepsilon}\right) \mathrm{d} x \\
& \quad+\int_{\Sigma^{\varepsilon}}\left(\mu^{\varepsilon} \circ \sigma\right)\left(B \nabla u^{\varepsilon}, \nabla u^{\varepsilon}\right)^{\frac{q}{2}-1}\left(B \nabla u^{\varepsilon}, \nabla\left(v-u^{\varepsilon}\right)\right) \mathrm{d} x \\
& \quad+\int_{\Sigma_{0}^{\varepsilon}}\left(\mu_{0}^{\varepsilon} \circ \sigma_{0}\right)\left(B_{0} \nabla u^{\varepsilon}, \nabla u^{\varepsilon}\right)^{\frac{q_{0}}{2}-1}\left(B_{0} \nabla u^{\varepsilon}, \nabla\left(v-u^{\varepsilon}\right)\right) \mathrm{d} x \\
& \quad-\int_{\Omega} f^{\varepsilon}\left(v-u^{\varepsilon}\right) \mathrm{d} x-\int_{\Sigma^{\varepsilon}} g^{\varepsilon}\left(v-u^{\varepsilon}\right) \mathrm{d} x-\int_{\Sigma_{0}^{\varepsilon}} g_{0}^{\varepsilon}\left(v-u^{\varepsilon}\right) \mathrm{d} x-I^{\varepsilon}\left(v-u^{\varepsilon}\right)_{\mid \Gamma^{\varepsilon}} \geqslant 0 .
\end{aligned}
$$

The solution $u$ of the limit problem $(\mathcal{P})$ is characterized by the variational formulation:

$$
u \in K, k \in \mathbb{R} u_{\mid \bar{\Gamma}} \in L^{q}(\bar{\Gamma}), u_{\mid \bar{\Gamma}_{0}} \in L^{q_{0}}\left(\bar{\Gamma}_{0}\right), u=k \text { on } \underline{\Gamma}, u=0 \text { on } \underline{\Gamma}_{0}
$$

and for any $v \in K, l \in \mathbb{R} v_{\mid \bar{\Gamma}} \in L^{q}(\bar{\Gamma}), v_{\mid \bar{\Gamma}_{0}} \in L^{q_{0}}\left(\bar{\Gamma}_{0}\right), v=l$ on $\underline{\Gamma}, v=0$ on $\underline{\Gamma}_{0}$,

$$
\begin{aligned}
& \int_{\Omega}(A \nabla u, \nabla u)^{\frac{p}{2}-1}(A \nabla u, \nabla(v-u)) \mathrm{d} x+\int_{\Omega} \phi(u)(v-u) \mathrm{d} x \\
& \quad+\int_{\bar{\Gamma}}(B n, n)^{1 / 2} a|u-k|^{q-2}(u-k)(v-l-u+k) \mathrm{d} s \\
& \quad+\int_{\bar{\Gamma}_{0}}\left(B_{0} n, n\right)^{1 / 2} a_{0}|u|^{q_{0}-2} u(v-u) \mathrm{d} s-\int_{\Omega} f(v-u) \mathrm{d} x-I(l-k) \geqslant 0 .
\end{aligned}
$$

For example, if $K=W^{1, p}(\Omega), u^{\varepsilon}$ is characterized by

$$
\begin{aligned}
& u^{\varepsilon} \in V^{\varepsilon} \text { and for every } v \in V^{\varepsilon} \\
& \int_{\Omega}\left(A \nabla u^{\varepsilon}, \nabla u^{\varepsilon}\right)^{\frac{p}{2}-1}\left(A \nabla u^{\varepsilon}, \nabla v\right) \mathrm{d} x+\int_{\Omega} \phi\left(u^{\varepsilon}\right) v \mathrm{~d} x \\
& \quad+\int_{\Sigma^{\varepsilon}}\left(\mu^{\varepsilon} \circ \sigma\right)\left(B \nabla u^{\varepsilon}, \nabla u^{\varepsilon}\right)^{\frac{q}{2}-1}\left(B \nabla u^{\varepsilon}, \nabla v\right) \mathrm{d} x
\end{aligned}
$$


M. BOUTKRIDA ET AL. / Ann. I. H. Poincaré - AN 19 (2002) 13-40

$$
\begin{aligned}
& +\int_{\Sigma_{0}^{\varepsilon}}\left(\mu_{0}^{\varepsilon} \circ \sigma_{0}\right)\left(B_{0} \nabla u^{\varepsilon}, \nabla u^{\varepsilon}\right)^{\frac{q_{0}}{2}-1}\left(B_{0} \nabla u^{\varepsilon}, \nabla v\right) \mathrm{d} x \\
& -\int_{\Omega} f^{\varepsilon} v \mathrm{~d} x-\int_{\Sigma^{\varepsilon}} g^{\varepsilon} v \mathrm{~d} x-\int_{\Sigma_{0}^{\varepsilon}} g_{0}^{\varepsilon} v \mathrm{~d} x-I^{\varepsilon} v_{\mid \Gamma^{\varepsilon}}=0
\end{aligned}
$$

and $u$ is characterized by:

$u \in W^{1, p}(\Omega), k \in \mathbb{R} u_{\mid \bar{\Gamma}} \in L^{q}(\bar{\Gamma}), u_{\mid \bar{\Gamma}_{0}} \in L^{q_{0}}\left(\bar{\Gamma}_{0}\right), u=k$ on $\underline{\Gamma}, u=0$ on $\underline{\Gamma}_{0}$

and for any $v \in W^{1, p}(\Omega), l \in \mathbb{R} v_{\mid \bar{\Gamma}} \in L^{q}(\bar{\Gamma}), v_{\mid \bar{\Gamma}_{0}} \in L^{q_{0}}\left(\bar{\Gamma}_{0}\right), v=l$ on $\underline{\Gamma}, v=0$ on $\underline{\Gamma}_{0}$,

$$
\begin{aligned}
\int_{\Omega}(A \nabla u, \nabla u)^{\frac{p}{2}-1}(A \nabla u, \nabla v) \mathrm{d} x+\int_{\Omega} \phi(u) v \mathrm{~d} x \\
+\int_{\bar{\Gamma}}(B n, n)^{1 / 2} a|u-k|^{q-2}(u-k)(v-l) \mathrm{d} s \\
+\int_{\frac{\Gamma_{0}}{}}\left(B_{0} n, n\right)^{1 / 2} a_{0}|u|^{q_{0}-2} u v \mathrm{~d} s-\int_{\Omega} f v \mathrm{~d} x-I l=0 .
\end{aligned}
$$

In other terms, $u^{\varepsilon}$ is a weak solution of

$$
\begin{cases}-\operatorname{div}\left(\left(A \nabla u^{\varepsilon}, \nabla u^{\varepsilon}\right)^{\frac{p}{2}-1} A \nabla u^{\varepsilon}\right)+\phi\left(u^{\varepsilon}\right)=f^{\varepsilon} & \text { in } \Omega, \\ -\operatorname{div}\left(\mu^{\varepsilon} \circ \sigma\left(B \nabla u^{\varepsilon}, \nabla u^{\varepsilon}\right)^{\frac{q}{2}-1} B \nabla u^{\varepsilon}\right)=g^{\varepsilon} & \text { in } \Sigma^{\varepsilon}, \\ -\operatorname{div}\left(\mu^{\varepsilon} \circ \sigma_{0}\left(B_{0} \nabla u^{\varepsilon}, \nabla u^{\varepsilon}\right)^{\frac{q_{0}}{2}-1} B_{0} \nabla u^{\varepsilon}\right)=g_{0}^{\varepsilon} & \text { in } \Sigma_{0}^{\varepsilon}, \\ \left(A \nabla u^{\varepsilon}, \nabla u^{\varepsilon}\right)^{\frac{p}{2}-1}\left(A \nabla u^{\varepsilon}, n\right)=0 & \text { on } \partial \Omega \backslash\left(\Gamma \cup \Gamma_{0}\right), \\ u^{\varepsilon} \text { is contant on } \Gamma^{\varepsilon}, & \\ \int_{\Gamma^{\varepsilon}}\left(\mu^{\varepsilon} \circ \sigma\right)\left(B \nabla u^{\varepsilon}, \nabla u^{\varepsilon}\right)^{\frac{q}{2}-1}\left(B \nabla u^{\varepsilon}, n\right) \mathrm{d} s=I^{\varepsilon}, & \\ u^{\varepsilon}=0 & \text { on } \Gamma_{0}^{\varepsilon}, \\ + \text { transmission conditions } & \text { on } \Gamma \cup \Gamma_{0},\end{cases}
$$

where the transmission conditions read

$$
\begin{cases}u_{\mid \Omega}^{\varepsilon}=u_{\mid \Sigma^{\varepsilon}}^{\varepsilon} \text { on } \Gamma, u_{\mid \Omega}^{\varepsilon}=u_{\mid \Sigma_{0}^{\varepsilon}}^{\varepsilon} & \text { on } \Gamma_{0}, \\ \left(A \nabla u_{\mid \Omega}^{\varepsilon}, \nabla u_{\mid \Omega}^{\varepsilon}\right)^{\frac{p}{2}-1}\left(A \nabla u_{\mid \Omega}^{\varepsilon}, n\right) & \\ \quad=\left(\mu^{\varepsilon} \circ \sigma\right)\left(B \nabla u_{\mid \Sigma^{\varepsilon}}^{\varepsilon}, \nabla u_{\mid \Sigma^{\varepsilon}}^{\varepsilon}\right)^{\frac{q}{2}-1}\left(B \nabla u_{\mid \Sigma^{\varepsilon}}^{\varepsilon}, n\right) & \text { on } \Gamma, \\ \left(A \nabla u_{\mid \Omega}^{\varepsilon}, \nabla u_{\mid \Omega}^{\varepsilon}\right)^{\frac{p}{2}-1}\left(A \nabla u_{\mid \Omega}^{\varepsilon}, n\right) & \\ \quad=\left(\mu_{0}^{\varepsilon} \circ \sigma_{0}\right)\left(B_{0} \nabla u_{\mid \Sigma_{0}^{\varepsilon}}^{\varepsilon}, \nabla u_{\mid \Sigma_{0}^{\varepsilon}}^{\varepsilon}\right)^{\frac{q_{0}}{2}-1}\left(B_{0} \nabla u_{\mid \Sigma_{0}^{\varepsilon}}^{\varepsilon}, n\right) & \text { on } \Gamma_{0} .\end{cases}
$$


Moreover the limit $u$ of $u^{\varepsilon}$ is a weak solution of

$$
\begin{cases}-\operatorname{div}\left((A \nabla u, \nabla u)^{\frac{p}{2}-1} A \nabla u\right)+\phi(u)=f & \text { in } \Omega, \\ (A \nabla u, \nabla u)^{\frac{p}{2}-1}(A \nabla u, n)=0 & \text { on } \Omega \backslash \backslash\left(\Gamma \cup \Gamma_{0}\right), \\ u=k, \text { undetermined constant, } & \text { on } \underline{\Gamma}, \\ (A \nabla u, \nabla u)^{\frac{p}{2}-1}(A \nabla u, n)+(B n, n)^{1 / 2} a|u-k|^{q-2}(u-k)=0 & \text { on } \bar{\Gamma}, \\ \int_{\Gamma}(A \nabla u, \nabla u)^{\frac{p}{2}-1}(A \nabla u, n) \mathrm{d} s=I, & \\ u=0 & \text { on } \underline{\Gamma}_{0}, \\ (A \nabla u, \nabla u)^{\frac{p}{2}-1}(A \nabla u, n)+\left(B_{0} n, n\right)^{1 / 2} a_{0}|u|^{q_{0}-2} u=0 & \text { on } \bar{\Gamma}_{0} .\end{cases}
$$

\section{REFERENCES}

[1] Acerbi E., Buttazzo G., Reinforcement problems in the calculus of variations, Ann. Inst. Henri Poincaré 3 (4) (1986) 273-284.

[2] Attouch H., Variational Convergence for Functions and Operators, Pitman, London, 1984.

[3] Boutkrida M., Doctoral Thesis, Ecole Normale Supérieure de Cachan, France, 1999.

[4] Boutkrida M., Mossino J., Moussa G., On nonhomogeneous reinforcements of varying shape and different exponents, Bolletino U.M.I. 2-B (8) (1999) 517-536. See also C. R. Acad. Sci. Paris, Série I 235 (1997) 565-570.

[5] Boutkrida M., Mossino J., Moussa G., On the torsional rigidity problem with nonhomogeneous reinforcement, Ricerche di Matematica (Supplemento) XLVIII (1999) 1-24.

[6] Brezis H., Caffarelli L.A., Friedman A., Reinforcement problems for elliptic equations and variational inequalities, Ann. Mat. Pura Appl. 123 (4) (1980) 219-246.

[7] Buttazzo G., Kohn R.V., Reinforcement by a thin layer with oscillating thickness, Appl. Math. Optim. 16 (1987) 247-261.

[8] Buttazzo G., Dal Maso G., Mosco U., Asymptotic behaviour for Dirichlet problems in domains bounded by thin layers, in: Essays in Honor of Ennio De Giorgi, Birkhaüser, Boston, 1989, pp. 193-249.

[9] Dal Maso G., An Introduction to Г-convergence, Birkhäuser, Boston, 1993.

[10] De Giorgi E., Convergence problems for functionals and operators, in: De Giorgi E., Magenes E., Mosco U., Lions J.L. (Eds.), Proc. "Recent Methods in Nonlinear Analysis", Rome, 1978, Pitagora, Bologna, 1979, pp. 131-188.

[11] Mossino J., Vanninathan M., Reinforcement of a multiconnected domain by a thin layer of oscillating thickness, to appear.

[12] Moussa G., Doctoral Thesis, Ecole Normale Supérieure de Cachan, France, 1998.

[13] Sanchez-Palencia E., Problèmes de perturbations liés aux phénomènes de conduction à travers des couches minces de grande résistivité, J. Math. Pures Appl. 53 (9) (1974) 251269.

[14] Sanchez-Palencia E., Nonhomogeneous Media and Vibration Theory, Lectures Notes in Physics, Springer Verlag, Berlin, 1980. 